\title{
EARLY DEVELOPMENT OF THE CEPHALIC SKELETON IN THE TURBOT
}

\author{
F. Wagemans ${ }^{\star}$, B. Focant $\dagger$ and P. Vandewalle* \\ *Institut de Zoologie, Laboratoire de Morphologie fonctionnelle, Université de Liège, \\ 22 Quai Van Beneden, 4020 Liège, Belgium \\ †Institut d'Anatomie, Laboratoire de Biologie cellulaire et tissulaire, Université de Liège, 20 rue des Pitteurs, \\ 4020 Liège, Belgium
}

At hatching Scophthalmus maximus shows no cartilaginous and no bony structure. Meckel's cartilages appear when the fry are 1 day old, followed on day 2, by formation of the trabecular bars, fused at the outset to form a trabecula communis. Concurrently, the palatoquadrates complete the mandibular arch, and the first two pairs of Ceratobranchials, associated with a pair of hyoid bars, form the beginnings of the hyobranchial system. By day 3, the parachordals have fused with the trabecular bars, the hyosymplectics have linked to the hyoid bars by interhyals, and the first four pairs of Ceratobranchials have appeared. The first bony structures appear: the preoperculars. On day 8 , the frontals develop above the orbits and the maxillaries and dentaries appear. On day 10, the primordia of the taeniae marginales appear, the palatoquadrates bear a pterygoid process, and to the branchial basket have been added the fifth pair of Ceratobranchials and the four pairs of epibranchials. On day 12, both pairs of posterior Pharyngobranchials are present. The premaxillaries develop in front of the maxillaires, and Tetroarticulars and the angulars complete the lower jaws. On day 13, a thin parasphenoid contributes to the floor of the neurocranium, and ectopterygoids and entopterygoids to the Splanchnocranium. The set of opercular bones is complete. On day 15, the tectum synoticum closes the braincase posteriorly. The Splanchnocranium possesses a basihyal and the Pharyngobranchials of the first epibranchials. On day 18 , the tectum posterius completes the dome of the braincase. The rear end and lateral walls of the skull are formed by the basioccipital, the exoccipitals, the pterotics, and the parietals. The suspensorium is nearly complete. From day 10, the first resorptions begin in parallel with the construction of the chondrocranium. Meckel's cartilages each split in two, then the posterior part of the trabecular bars disappears. On day 23, the right taenia marginalis separates from the lamina orbitonasalis and curves towards the centre. Simultaneously, the right eye begins its migration to the left. This is the only metamorphosislinked asymmetry to appear during the development of the chondrocranium. On day 25, many more bony structures appear, a characteristic of this stage: the nasals, lateral ethmoids, mesethmoid, sphenotics, prootics, pleurosphenoids, epiotics, and Supraoccipital. From this stage on, the bony structures continue to develop, while the front of the neurocranium and the jaws undergo a deep remodelling due to metamorphosis. The left taenia marginalis does not appear reduced until day 29 . By day 45 , there remain only a few small elements of the cartilaginous skull.

KEYWORDS: postembryonic development; skeleton; Pleuronectiforms; metamorphosis; Scophthalmidae; Scophthalmus. 


\section{INTRODUCTION}

For many years, scientists have been interested in the postembryonic development [the eleutheroembryonic and larval stages according to Krupka (1988)] of the cephalic skeleton of teleost fish (Parker, 1873; Stohr, 1882; Tischomiroff, 1885; Winslow, 1897; Swinnerton, 1902; Gaupp, 1903; Bamford, 1948; Bertmar, 1959; Daget, 1964; Jollie, 1975; Badenhorst, 1989a,b; Kobayakawa, 1992; Vandewalle et al., 1995 $\alpha$ ). There have been very few detailed studies, however, concerning the development of the skull in pleuronectiforms from hatching (day 0 ) to the juvenile stage. Published accounts usually either give an external description of the different stages in the development of flatfish fry, with emphasis on the characteristics of pleuronectiforms, or describe anomalies in the cephalic area of laboratory-reared larvae (Blaxter et al., 1983; Sadiq \& Gibson, 1984; Liew et al., 1988; Lagardère, 1993).

At hatching, pleuronectiform larvae are good swimmers and have the principal external morphological characteristics of other teleost larvae; the eyes and nostrils are present on both sides of the head, the mouth is horizontal or subhorizontal, and the fins are usually in place. During development, one eye migrates to the contralateral side of the head and the whole head undergoes profound modification.

The larvae start to swim on their sides and finally adopt a benthic life. This metamorphosis, which concerns essentially the cephalic region, must characteristically affect how the skeletal elements develop. The morphological changes associated with the transition from a pelagic life to a benthic one must have consequences on the feeding mechanism. For example, one feature that must reflect this change is the orientation of the mouth in relation to the prey.

Turbot Scophthalmus maximus (L.) is a pleuronectiform with sinistral metamorphosis. Their larvae are planktonophages, while the adults feed on crustaceans, bivalve molluscs, and fish. This study describes the timing of events connected with the development of the cephalic skeleton before, during, and after metamorphosis. Detailed knowledge of these events should contribute to a better understanding of the feeding mechanisms that are essential to fry survival at every stage of development. The nomenclature used to describe the skeletal structures is based principally on the works of De Beer (1937) and Daget (1964). 


\section{MATERIALS AND METHODS}

S. maximus fry were reared at $15^{\circ} \mathrm{C}$ on the France Turbot-NATA fish farm (Noirmoutier, France). Samples of 30 fry were taken at intervals from hatching up to day 57 following. The fry were fixed in a $\mathrm{CaCO}_{3}$-buffered $10 \%$ formalin solution, cleared with trypsin and stained with either alcyan blue (to reveal the cartilages) or alizarin (to reveal the bones). The staining techniques were derived from those of Dingerkus \& Uhler (1977), Potthoff (1983) and Taylor \& van Dyke (1985).

The drawings were produced by means of a camera lucida mounted on a Wild M10 Leica binocular.

Although the presentation of the results begins with fry that have just hatched, homologies were established and structures recognized only by comparing the structures found in adult fish with those of older fry, then the structures of these older fry with those of younger ones, and so on to the youngest fry.

\section{RESULTS}

\section{DAY 0 (HATCHING)}

No cephalic skeletal structure is visible.

\section{DAY 1}

CHONDROCRANIUM [FIG. 1(A)]

Meckel's cartilage, i.e. the lower part of the mandibular arch, is present as a simple straight bar.

FIG. 1. Scophthalmus maximus:

(a)

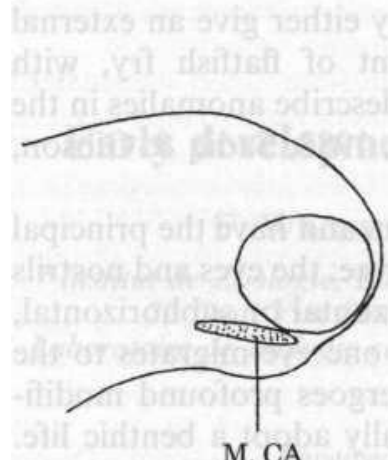

M. CA (b)
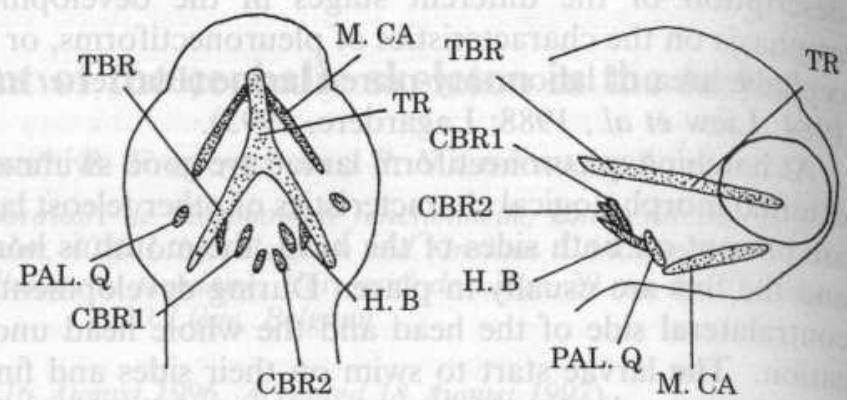

(c)

M. CA

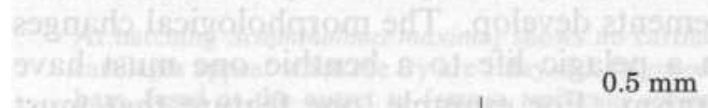


(a) lateral view of the chondrocranium of a 1-day-old fry; dorsal (b) and lateral (c) views of the chondrocranium of a 2day-old fry. CBR, Ceratobranchial; H. B, hyoid bar; M. CA, Meckel's cartilage; PAL. Q, palatoquadrate; TR, trabecula communis; TRB, trabecular bar.

\section{DAY 2}

\section{CHONDROCRANIUM [FIG. 1(B) AND (C) ]}

Several cartilaginous elements have appeared: the trabecular bars, which are the first parts of the neurocranium, are joined anteriorly at the outset to form the start of the trabecular communis, a part of each palatoquadrate contributes to the mandibular arch, and three pairs of elements belonging to the next visceral arches form the start of the hyobranchial system. These latter elements are either one pair of hyoid bars and two pairs of Ceratobranchials (Fig. 1(b) and (c)], or three pairs of Ceratobranchials.

\section{DAY 3}

\section{CHONDROCRANIUM [FIG. 2(A), (B) AND (C)]}

At the front of the neurocranium, the trabecular communis has widened into an ethmoid plate, and at the back, the parachordal plates have appeared already fused with the small-scale trabecular bars. Each parachordal plate displays a lateral extension, which is probably the start of the commissura basicapsularis anterior. The hyoid arch is represented by two well-developed hyosymplectics, particularly wide in their upper part and in contact with the palatoquadrates at their lower ends. The hyosymplectics in their upper anterior part display a foramen, the truncus hyoideomandibularis, which is present throughout development. A pair of independent interhyals is present between the hyosymplectics and the hyoid bars. The branchial basket is formed by four pairs of Ceratobranchials and a long, median basibranchial bar.

\section{OSTEOCRANIUM [FIG. 2(C)]}

No stained bony element is visible but the outline of a small preopercular can be seen on each side of the head. Two alcyan-blue-stained skin folds are present, one on each side of the head, at the edge of the upper lip. 
FIG. 2. Scophthalmus maximus:

(a)

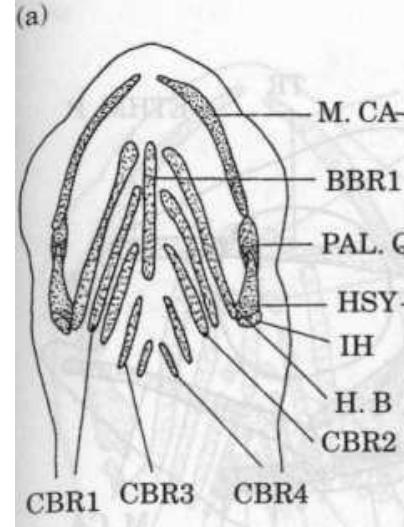

(b)

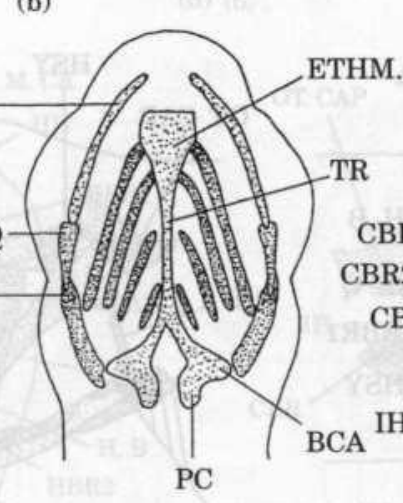

(c)

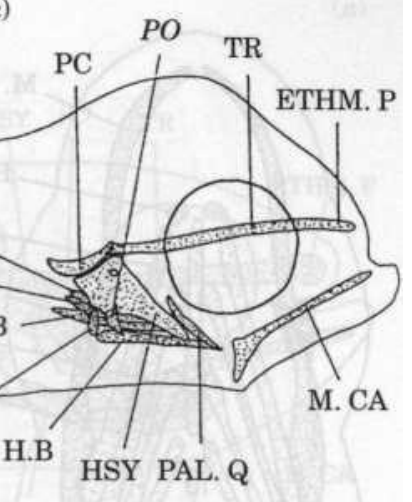

ventral (a) dorsal (b) and lateral (c) views of the chondrocranium and osteocranium of a 3-day-old fry. BBR1, Basibranchial 1-3; BCA, commissura basicapsularis anterior; CBR, Ceratobranchial; ETHM. P, ethmoid plate; H. B, hyoid bar; HSY, hyosymplectic; IH, interhyal; M. CA, Meckel's cartilage; PAL. Q, palatoquadrate; PC, parachordal plate; PO, preopercular; TR, trabecula communis. Abbreviations in italic show unstained bony structures.

\section{FIG. 3. Scophthalmus maximus:}

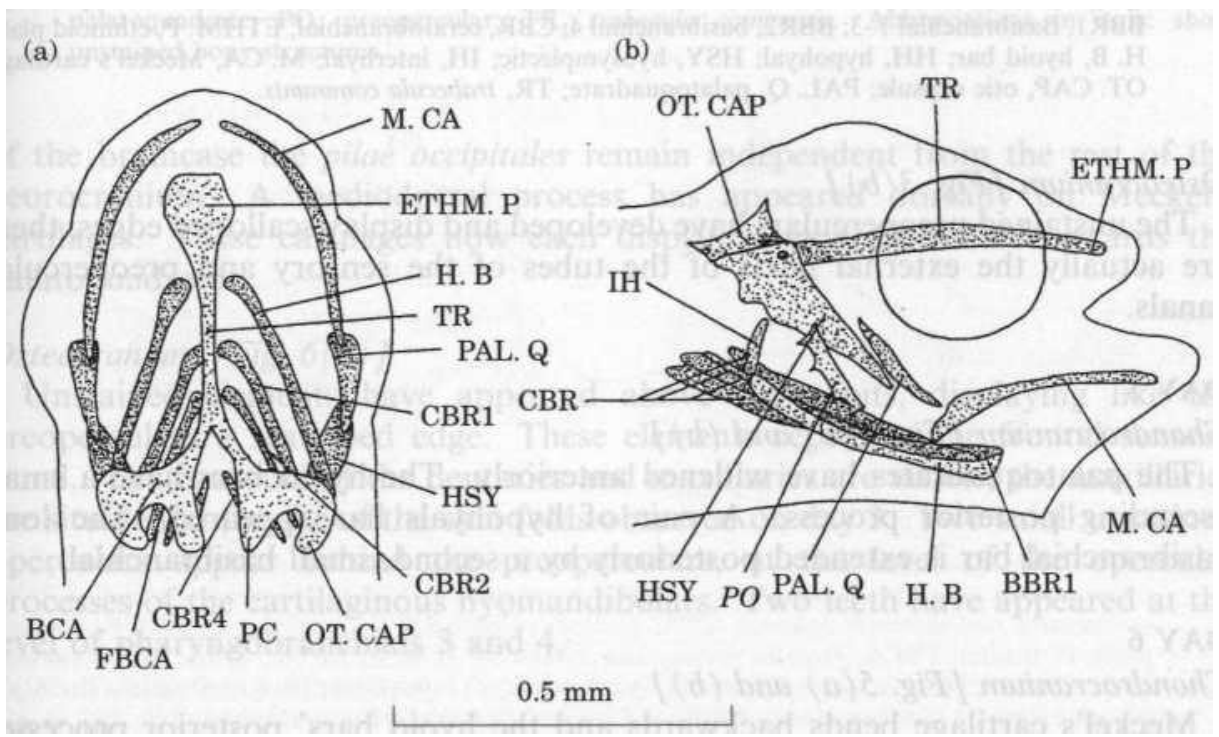

dorsal (a) and lateral (b) views of the chondrocranium and osteocranium of a 4-day-old fry. BBR1, Basibranchial 1-3; BCA, commissura basicapsularis anterior; CBR, Ceratobranchial; ETHM. P, ethmoid plate; FBCA, fenestra basicapsularis anterior; H. B. hyoid bar; HSY, hyosymplectic; IH, interhyal; M. CA, Meckel's cartilage; OT. CAP, otic capsule; PAL. Q, palatoquadrate; PC, parachordal plate; PO, preopercular; TR, trabecula communis. Abbreviations in italic show unstained bony structures. 


\section{DAY 4}

\section{CHONDROCRANIUM [FIG. 3(A) AND (B)]}

The otic capsules are developing and are joined directly to the parachordal plates by the commissura basicapsularis anterior.

FIG. 4. Scophthalmus maximus:

(a)

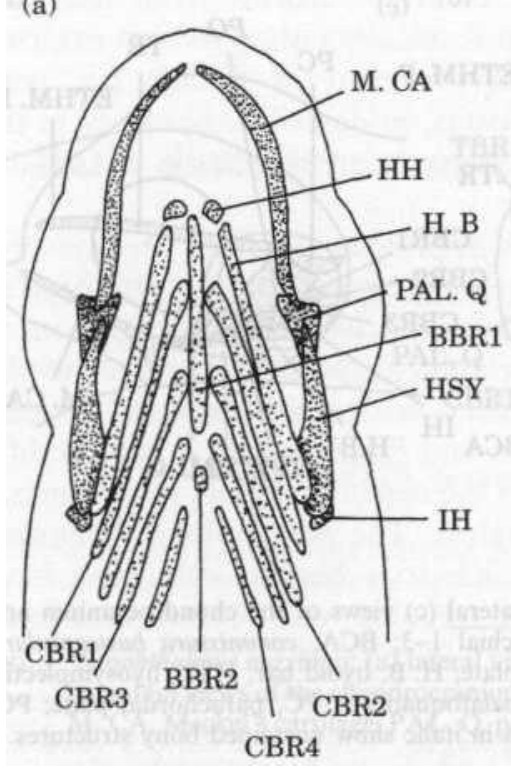

(b)

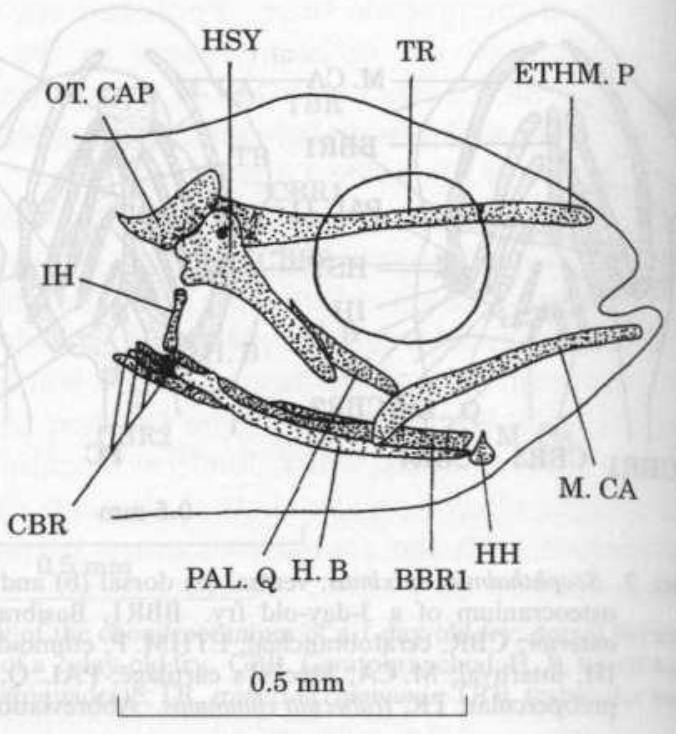

ventral (a) and lateral (b) views of the chondrocranium of a 5-day-old fry. BBR1, Basibranchial 1-3; BBR2, basibranchial 4; CBR, Ceratobranchial; ETHM. P, ethmoid plate; H. B, hyoid bar; HH, hypohyal; HSY, hyosymplectic; IH, interhyal; M. CA, Meckel's cartilage; OT. CAP, otic capsule; PAL. Q, palatoquadrate; TR, trabecula communis.

\section{OSTEOCRANIUM [ FIG. 3(B) ]}

The unstained preoperculars have developed and display scalloped edges; these are actually the external parts of the tubes of the sensory and preopercular canals.

\section{DAY 5}

\section{CHONDROCRANIUM [FIG. 4(A) AND (B)]}

The palatoquadrates have widened anteriorly. The hyoid bars have a small ascending posterior process. A pair of hypohyals has appeared. The long basibranchial bar is extended posteriorly by a second, small basibranchial. 


\section{DAY 6}

\section{CHONDROCRANIUM [FIG. 5(A) AND (B) ]}

Meckel's cartilage bends backwards and the hyoid bars' posterior processes have grown larger. Three pairs of hypobranchials have appeared in the branchial basket, splitting off from Ceratobranchials 1, 2, and 3 .

OSTEOCRANIUM [FIG. 5(B)]

The unstained preoperculars are still growing.

\section{DAY 8}

\section{CHONDROCRANIUM [FIG. 6(A) AND (B)]}

The parachordal plates, otic capsules, and commissurae basicapsulares anteriores surround almost completely the fenestrae basicapsulares. At the back of the braincase the pilae occipitales remain independent from the rest of the neurocranium. A mediodorsal process has appeared dorsally on Meckel's cartilages. These cartilages now each display a socket oriented towards the palatoquadrate.

FIG. 5. Scophthalmus maximus:

(a)

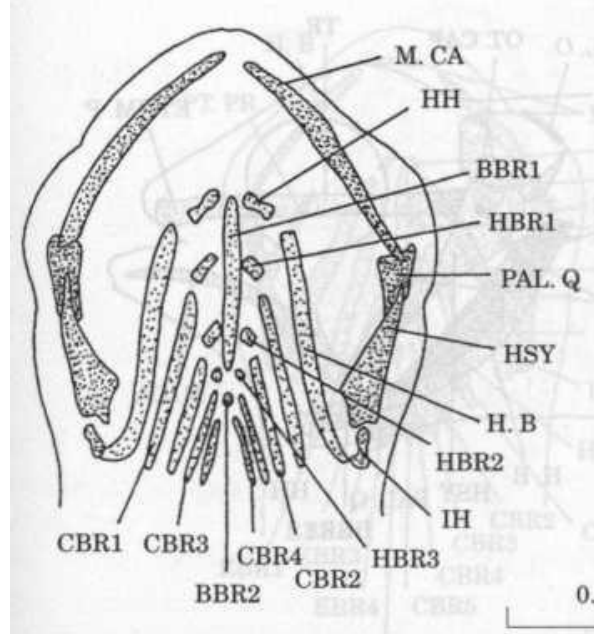

(b)

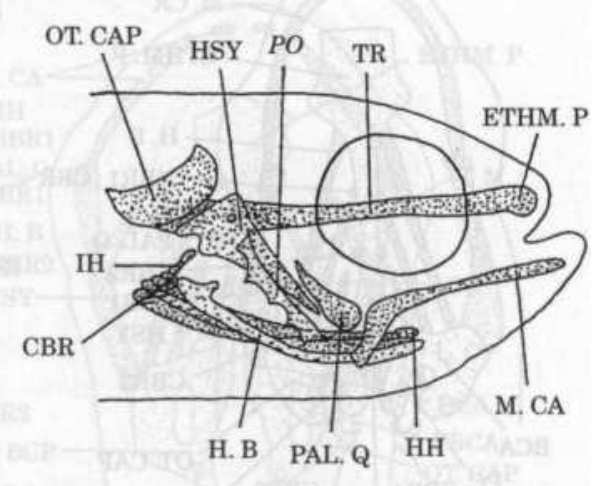

$0.5 \mathrm{~mm}$

ventral (a) lateral (b) views of the chondrocranium and OSteocranium of a 6-day-old fry. BBR1, Basibranchial 1-3; BBR2, basibranchial 4; CBR, Ceratobranchial; ETHM. P, ethmoid plate; H. B, hyoid bar; HBR, hypobranchial; HH, hypohyal; HSY, hyosymplectic; IH, interhyal; M. CA, Meckel's cartilage; OT. CAP, otic capsule; PAL. Q, palatoquadrate; PO, preopercular; TR, trabecula communis. Abbreviations in italic show unstained bony structures. 


\section{OSTEOCRANIUM [FIG. 6(C)]}

Unstained elements have appeared above the orbits, displaying like the preoperculars a scalloped edge. These elements represent the frontal sensory canal. The contours of the dentaries and maxillaries are visible, the maxillaries are situated at the site of the skin folds observed on day 3. Two small unstained operculars appear behind the preoperculars, at the level of the opercular processes of the cartilaginous hyomandibulars. Two teeth have appeared at the level of pharyngobranchials 3 and 4 .

\section{FIG. 6. Scophthalmus maximus}

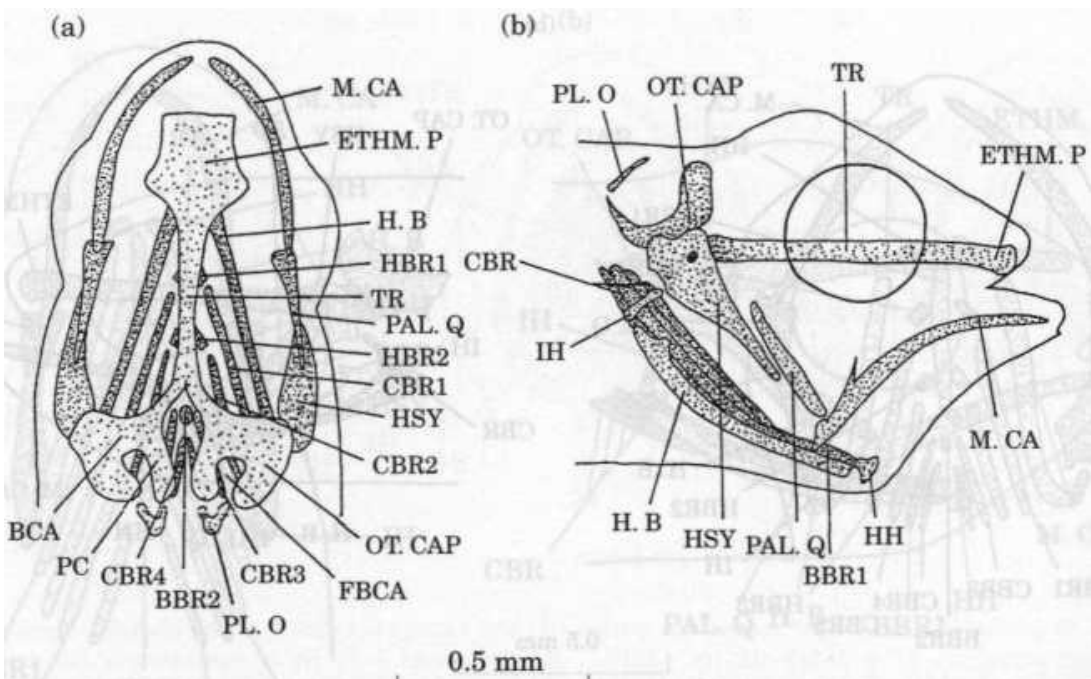

(c)

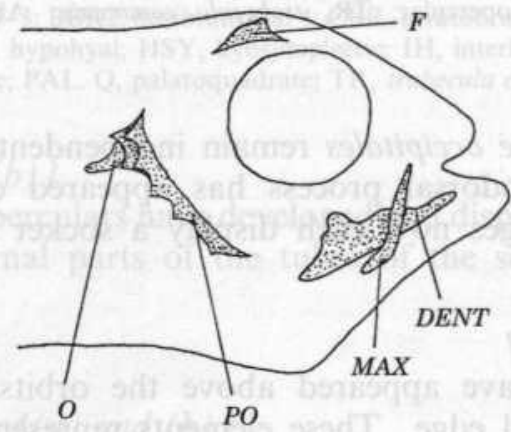

$0.5 \mathrm{~mm}$

dorsal (a) and lateral (b) views of the chondrocranium, lateral view of the osteocranium (c) of an 8-day-old fry. BBR1, basibranchial 1-3; BBR2, basibranchial 4; BCA, commissura basicapsularis anterior; CBR, Ceratobranchial; DENT, dentary; ETHM. P, ethmoid plate; F, frontal; FBCA, fenestra basicapsularis anterior; H. B, hyoid bar; HBR, hypobranchial; HH, hypohyal; HSY, hyosymplectic; IH, interhyal; MAX, maxillary; M. CA, Meckel's cartilage; O, opercular; OT. CAP, otic capsule; PAL. Q, palatoquadrate; PC, parachordal plate; PL. O, pila occipitalis; PO, preopercular; TR, trabecula communis. Abbreviations in italic show unstained bony structures. 


\section{DAY 10}

\section{CHONDROCRANIUM [FIG. 7(A), (B) AND (C)]}

The taeniae marginales have appeared dorsally in the neurocranium. At the back of the trabecular communis a cartilaginous column has appeared on each side, linking it to the front and top of the otic capsule. These are probably the commissurae laterales. The backs of the lateral walls of the otic capsules are in contact with the parachordal plates, probably via the commissura basicapsularis posterior, thereby defining a large fenestra basicapsularis. At the back of the braincase, the pilae occipitales touch the parachordal plates and the otic capsules, probably defining the foramen of the vagus nerve. Each palatoquadrate is extended by the beginnings of a pterygoid process. The branchial basket now includes the fifth pair of Ceratobranchials and the four pairs of epibranchials. Meckel's cartilages have regressed anteriorly, isolating two small cartilaginous lumps. The posterior part of each hyoid bar has flattened and broadened.

\section{OSTEOCRANIUM [FIG. 7(D)]}

The unstained elements above the orbits have enlarged and each consists of two parts: an inner part with a smooth edge and a scalloped outer part: these are the frontals, each with the tube of its sensory canal. The operculars have developed and the tooth plates, each bearing at least two teeth, have formed at the level of pharyngobranchials 3 and 4 .

FIG. 7. Scophthalmus maximus:

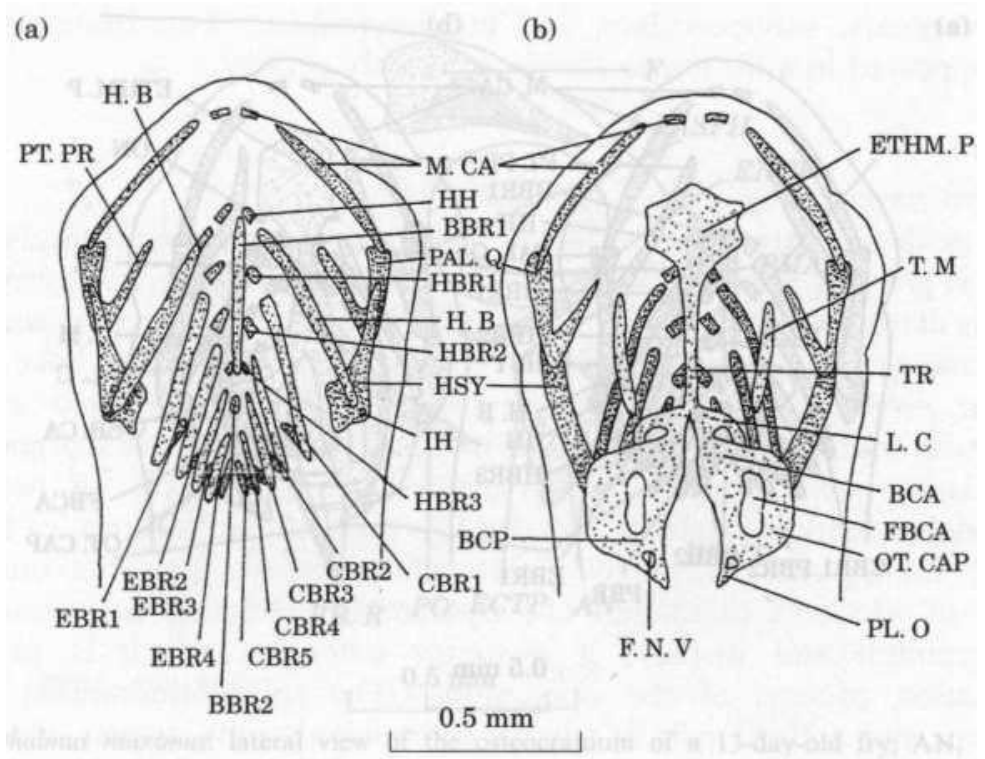


(c)

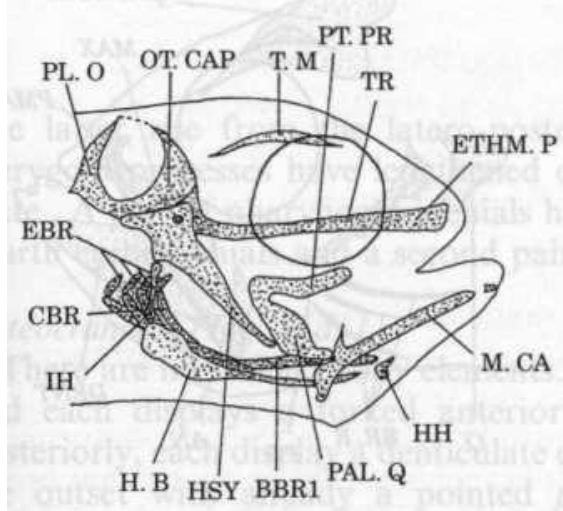

(d)

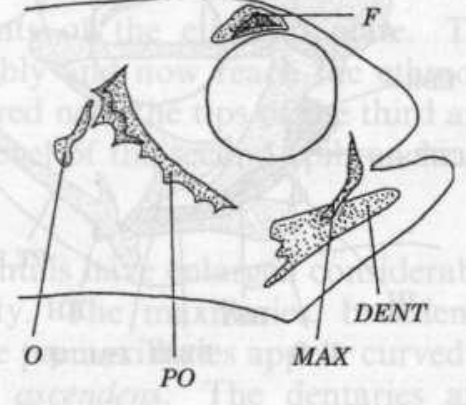

$0.5 \mathrm{~mm}$

ventral (a) dorsal (b) and lateral (c) views of the chondrocranium, lateral iew of the osteocranium (d) of a 10-day-old fry. BBR1, basibranchial 1-3; BBR2, basibranchial 4; BCA, commissura basicapsularis anterior; BCP, commissura basicapsularis posterior: CBR, Ceratobranchial; DENT, dentary; EBR. epibranchial; ETHM. P, ethmoid plate; F, frontal; F. N. V., foramen for the vagus nerve; H. B, hyoid bar; HBR. hypobranchial; HH, hypohyal; HSY, hyosymplectic; IH, interhyal; L. C, commissura lateralis: MAX, maxillary; M. CA, Meckel's cartilage; O, opercular; OT. CAP, otic capsule; PAL. Q, palatoquadrate; PL. O, pila occipitalis: PO, preopercular; PT. PR, pterygoid process; TM, taenia marginalis: TR, trabecula communis. Abbreviations in italic show unstained bony structures.

\section{DAY 12}

\section{CHONDROCRANIUM [FIG. 8(A), (B) AND (C)[}

Posteriorly, the taeniae marginales are continuous with the laterally closed otic capsules; anteriorly, they extend in the direction of the laminae Orbitonasales. The latter rise from the latero-posterior points of the ethmoid plate. The pterygoid processes have lengthened considerably and now reach the ethmoid plate. A pair of pharyngobranchials has appeared near the tips of the third and fourth epibranchials and a second pair at the level of the second epibranchials.

\section{OSTEOCRANIUM [FIG. 8(D)]}

There are no stained bony elements. The frontals have enlarged considerably and each displays a forked anterior extremity. The maxillaries, broadened posteriorly, each display a denticulate edge. The premaxillaries appear curved at the outset with already a pointed processus ascendens. The dentaries and angulars, closely applied but distinct from each other, constitute the lower jaws whose shape already prefigures the jaws of the future adult. The coronoid processes of the dentaries and angulars are clearly recognizable. Tooth plates have appeared on the fifth pair of cartilaginous Ceratobranchials: these are the first bony primordia of the lower pharyngeal jaws. A tooth plate has appeared below each cartilaginous second pharyngobranchial, an addition to the set of tooth plates already present at the previous stage. The whole set constitutes a rudimentary upper pharyngeal jaw. Three branchiostegal rays are present on each side of the head. 
FIG. 8. Scophthalmus maximus:

(a) (b)

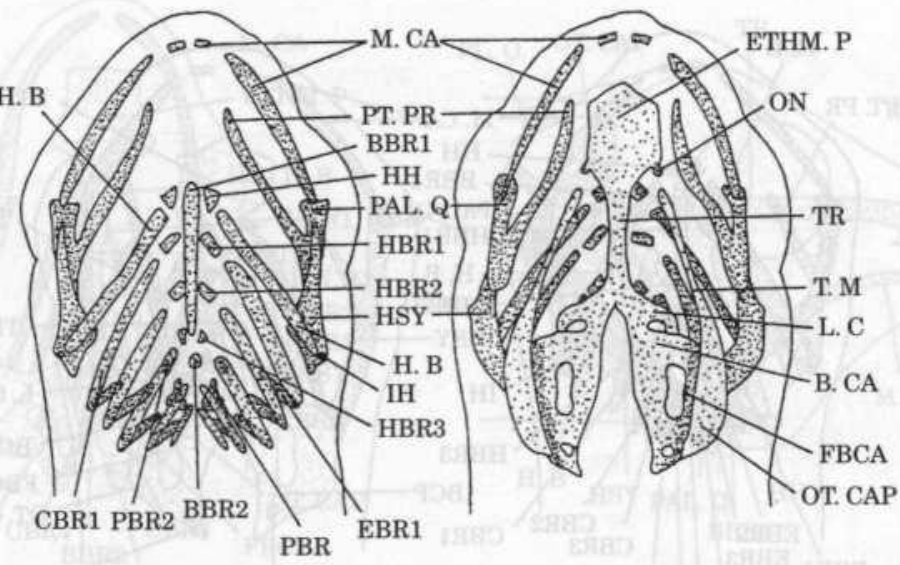

$0.5 \mathrm{~mm}$ (c)

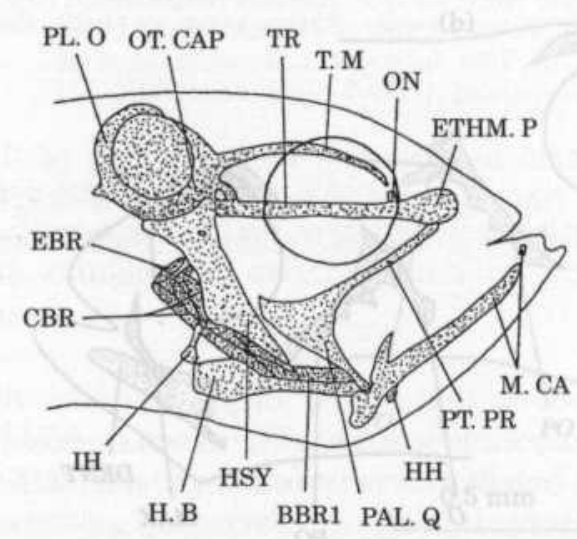

(d)

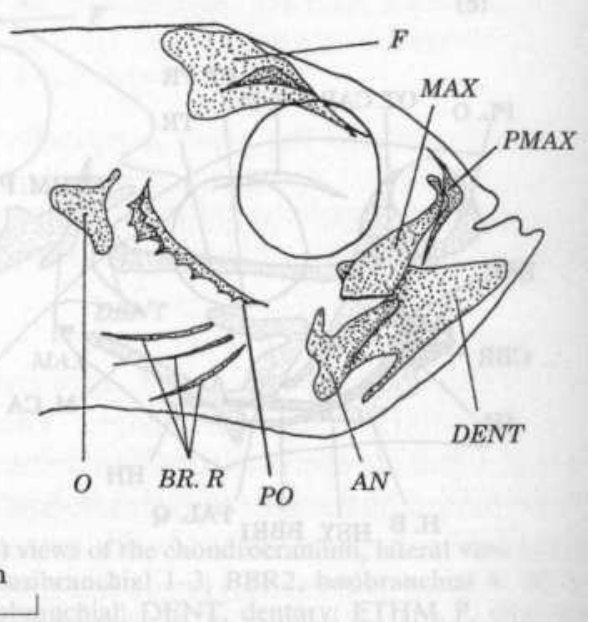

ventral (a) dorsal (b) and lateral (c) views of the chondrocranium, lateral view of the osteocranium (d) of a 12-day-old fry. AN, angular; BBR1. basibranchial 1-3; BBR2, basibranchial 4; BCA, commissura basicapsularis anterior; BR. R. branchiostegal rays; CBR. Ceratobranchial; DENT, dentary; EBR, epibranchial; ETHM. P, ethmoid plate; F, frontal; FBCA, fenestra basicapsularis anterior; H. B, hyoid bar; HBR, hypobranchial; $\mathrm{HH}$, hypohyal; HSY, hyosymplectic; IH, interhyal; L. C, commissura lateralis; MAX, maxillary; M. CA, Meckel's cartilage; O, opercular; ON, lamina Orbitonasalis; OT. CAP, otic capsule; PAL. Q, palatoquadrate; PBR, pharyngobranchial; PL. O, pila occipitalis; PMAX, premaxillary; PO, preopercular; PT. PR, pterygoid process; TM. taenia marginalis; TR, trabecula communis. Abbreviations in italic show unstained bony structures. 


\section{DAY 13}

\section{OSTEOCRANIUM (FIG. 9)}

There are no stained bony elements. A thin parasphenoid can be recognized between the orbits. The Splanchnocranium now possesses entopterygoids, ectopterygoids, Suboperculars, and interoperculars. Two branchiostegal rays have appeared in addition to the three already present.

FIG. 9. Scophthalmus maximus:

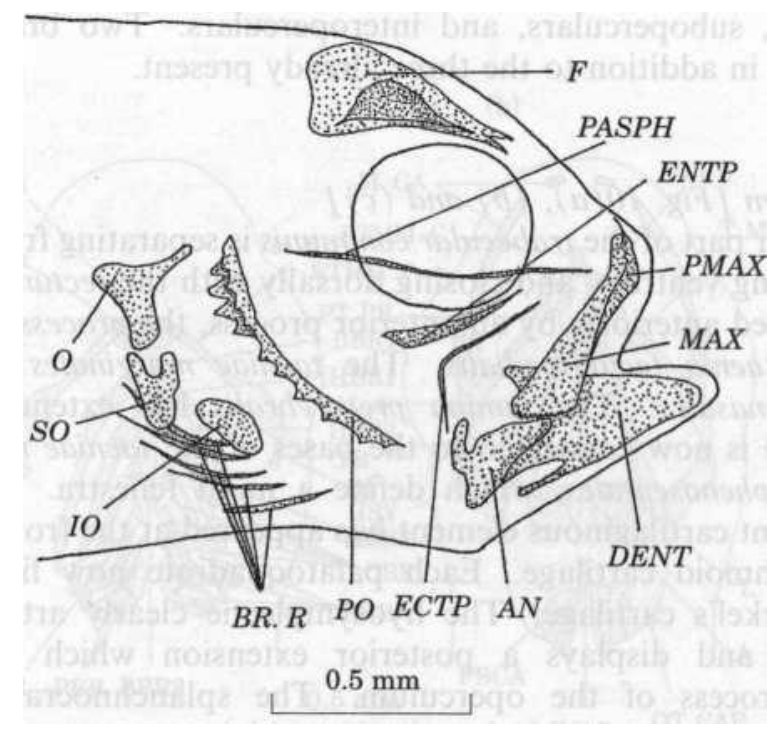

lateral view of the osteocranium of a 13-day-old fry; AN, angular; BR. R, branchiostegal rays; DENT, dentary; ECTP, ectopterygoid; ENTP, entopterygoid; F, frontal; IO, interopercular; MAX, maxillary; O, opercular; PASPH, parasphenoid; PMAX, premaxillary; PO, preopercular; SO, Subopercular. Abbreviations in italic show unstained bony structures.

\section{DAY 15}

\section{CHONDROCRANIUM [FIG. 10(A), (B) AND (C)]}

The posterior part of the trabecular communis is separating from the braincase, which is growing ventrally and closing dorsally with the tectum synoticum. The latter is extended anteriorly by an anterior process, the processus anterior of the braincase or taenia tecta medialis. The taeniae marginales have joined the laminae orbitonasales. The lamina precerebralis has extended upward and backward, and is now connected to the bases of the taeniae marginales by the commissurae sphenoseptales, which define a nasal fenestra. A new unpaired and independent cartilaginous element has appeared at the front of the ethmoid region: the ethmoid cartilage. Each palatoquadrate now fits into the joint socket of Meckel's cartilage.

The hyosymplectic clearly articulates with the neurocranium and displays a posterior extension which is probably the articulation process of the operculum. The Splanchnocranium displays a 
basihyal, and a third pair of pharyngobranchials now appear above the first epibranchials. The first, second, and third epibranchials each display an uncinate process.

\section{OSTEOCRANIUM [FIG. 10(D)]}

No bony element is stained. The basioccipital is now present in the neurocranium. The upper and lower jaws bear teeth. There are six pairs of branchiostegal rays.

FIG. 10. Scophthalmus maximus:

(a)

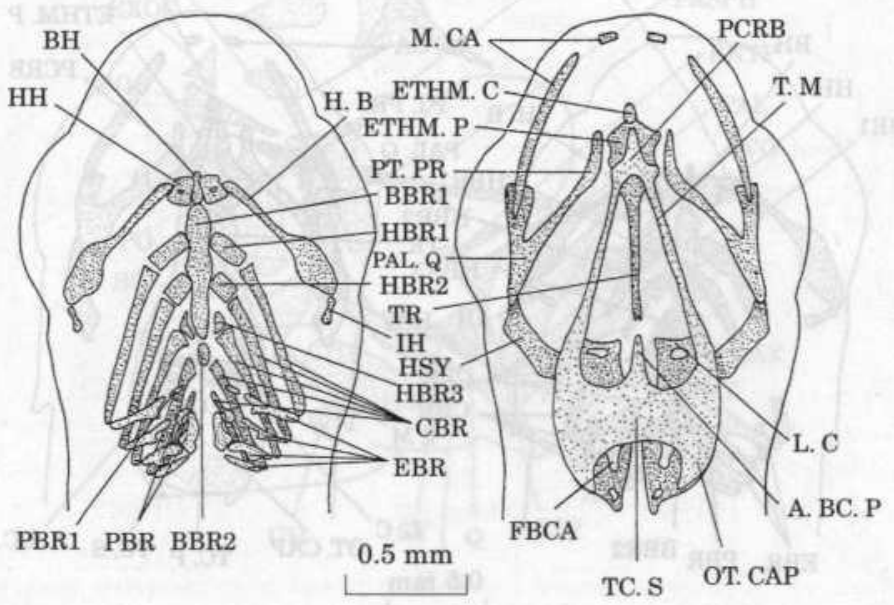

(b) (c)

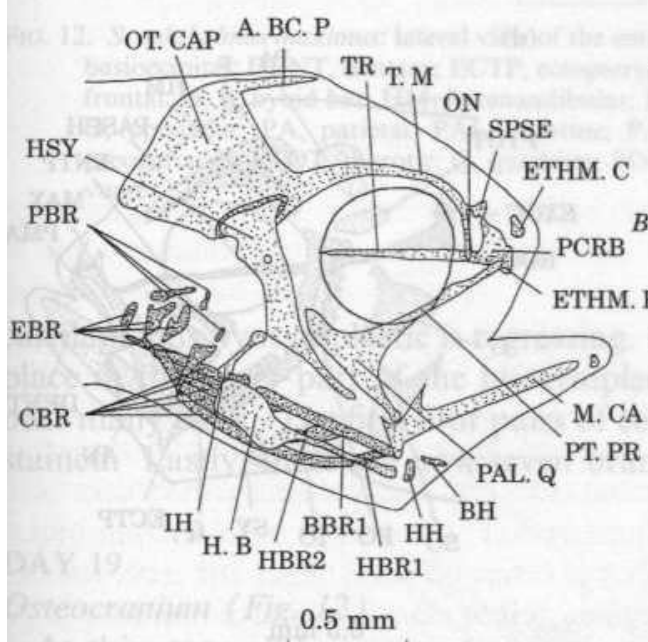

(d)

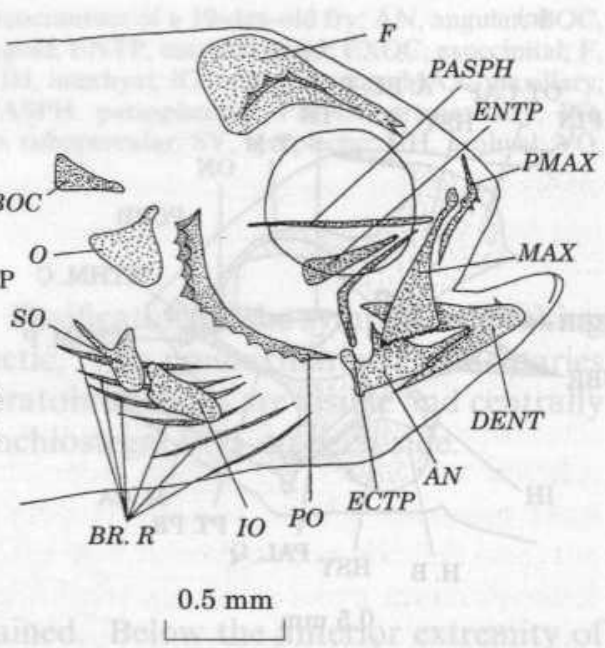

dorsal view of the branchial basket (a), dorsal (b) and lateral (c) views of the chondrocranium, lateral view of the osteocranium (d) of a 15-day-old fry; A. BC. P, anterior process of the braincase; AN, angular; BBR1, basibranchial 1-3; BBR2, basibranchial 4; BH, basihyal; BOC. basioccipital; BR. R, branchiostegal rays; CBR, Ceratobranchial; DENT, dentary; EBR, epibranchial; ECTP, ectopterygoid; ENTP, entopterygoid; ETHM. C. ethmoid cartilage; ETHM. P, ethmoid plate; F, frontal; FBCA, fenestra basicapsularis anterior; H. B, hyoid bar; HBR. hypobranchial; HH, hypohyal; HSY, hyosymplectic; IH. interhyal; IO. interopercular; L. C, commissura lateralis; MAX, maxillary; M. CA, Meckel's cartilage; O, opercular; ON, lamina orbitonasalis; OT. CAP, otic capsule; PAL. Q, palatoquadrate; PASPH, parasphenoid; PBR. pharyngobranchial; 
PCRB, lamina precerebralis; PL. O, pila occipitalis; PMAX, premaxillary; PO, preopercular; PT. PR, pterygoid process; SO, Subopercular; SPSE, commissura Sphenoseptalis; TC. S, tectum synocticum; TM, taenia marginalis; TR, trabecula communis. Abbreviations in italic show unstained bony structures.

\section{DAY 18}

\section{CHONDROCRANIUM [FIG. 11(A), (B) AND (C)]}

The dome of the braincase has been completed posteriorly by the tectum posterius, which links it to the pilae occipitales. The hyosymplectics are regressing and this isolates the joint region from the rest of the hyosymplectic. The nasal fenestra has become smaller. The central region of each Ceratobranchial is regressing, except for the fifth pair of Ceratobranchials. The hyoid bar has begun regressing at its centre (very weak staining of the chondroblasts by alcyan blue). The uncinate process of each third epibranchial has lengthened and forms a bridge towards the fourth epibranchials without touching them. The dorsal fin has appeared behind the otic capsule.

\section{OSTEOCRANIUM [FIG. 11(D)]}

The frontals, parasphenoid, and basioccipital are well stained. Two small unstained exoccipitals have appeared, one on each side of the basioccipital, along with two parietals and two weakly stained pterotics. The Splanchnocranium also exhibits stained elements: the premaxillaries, maxillaries, dentaries, angulars, ectopterygoids, entopterygoids, and the set of operculars. New stained elements have appeared: the hyomandibulars, symplectics, and quadrates. The ossified portion of each quadrate is the joint region in connection with the mandible. The ossified part of each hyomandibular corresponds with the still-cartilaginous joint region connected to the neurocranium and with the region where the cartilaginous hyosymplectic is regressing. Ossification of the Symplectic is taking place in the lower part of the hyosymplectic. The premaxillaries and dentaries bear many teeth. The first four pairs of Ceratobranchials are visible and centrally stained. Lastly, there are now seven branchiostegal rays on each side. 
FIG. 11. Scophthalmus maximus:

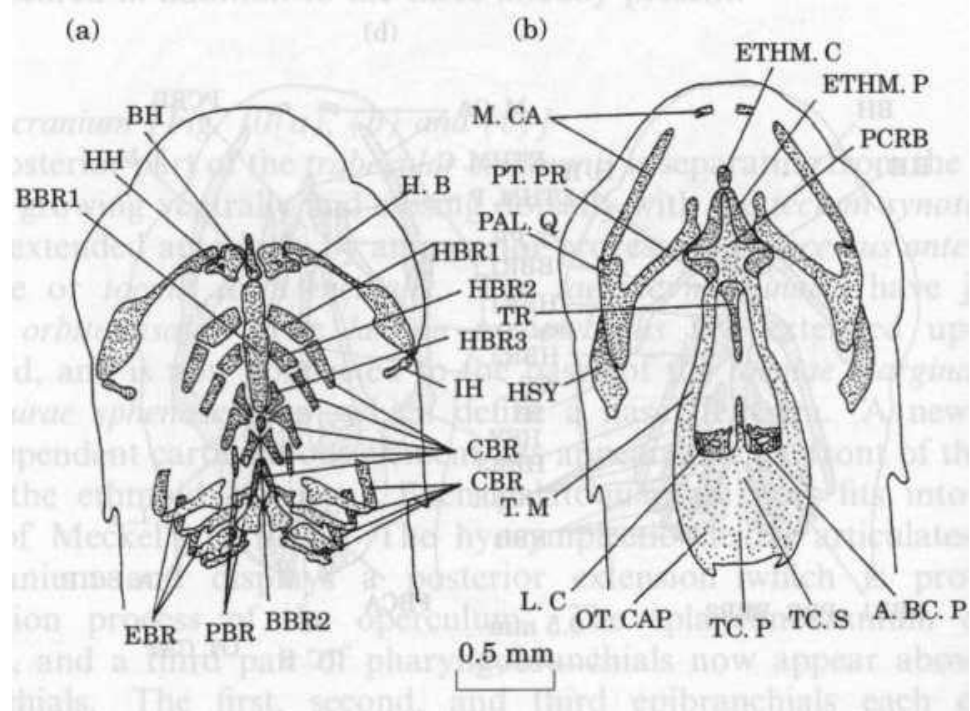

(c)

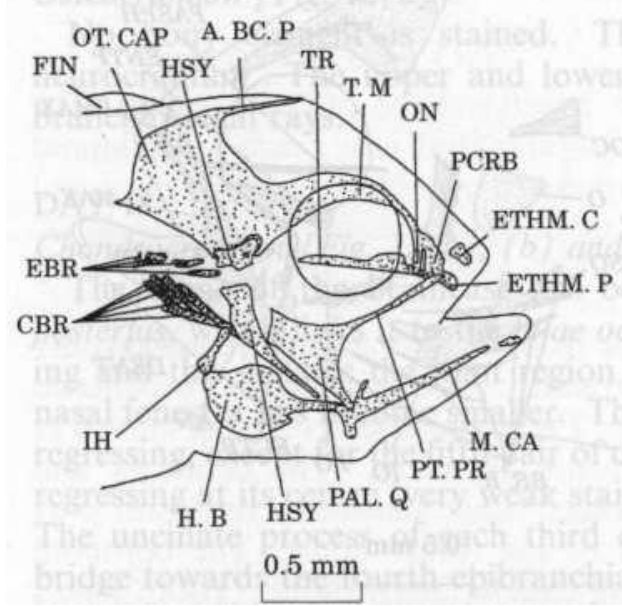

(d)

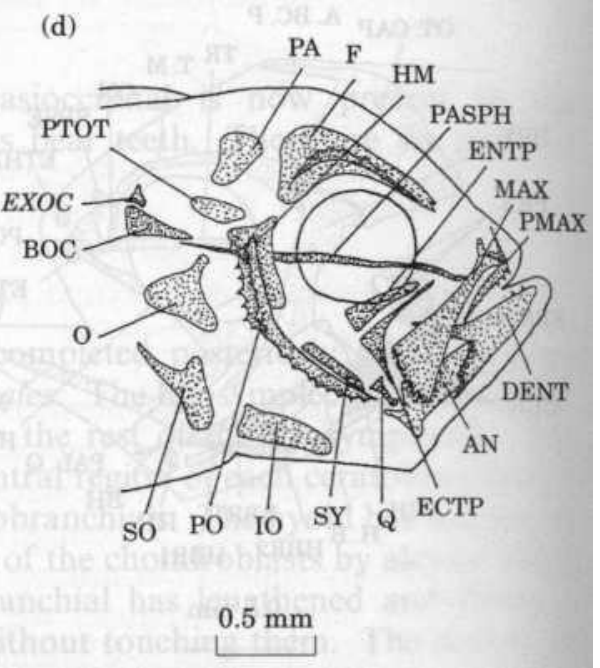

dorsal view of the branchial basket (a), dorsal (b) and lateral (c) views of the chondrocranium, lateral view of the OSteocranium (d) of an 18-day-old fry; A. BC. P. anterior process of the braincase; AN, angular; BBR1, basibranchial 1-3; BBR2, basibranchial 4; $B H$, basihyal; BOC, basioccipital; CBR, Ceratobranchial; DENT, dentary; EBR, epibranchial; ECTP, ectopterygoid; ENTP, entopterygoid; ETHM. C, ethmoid cartilage; ETHM. P, ethmoid plate; EXOC, exoccipital; F, frontal; H. B, hyoid bar; HBR, hypobranchial; HH, hypohyal; HM, hyomandibular; HSY, hyosymplectic; IH, interhyal; IO, interopercular; L. C, commissura lateralis: MAX, maxillary; M. CA, Meckel's cartilage; O, opercular; ON, lamina orbitonasalis: OT. CAP. otic capsule; PA, parietal; PAL. Q, palatoquadrate; PASPH, parasphenoid; PBR. pharyngobranchial; PCRB, lamina precerebralis; PMAX, premaxillary; PO, preopercular; PTOT. pterotic; PT. PR, pterygoid process; Q, quadrate; SO, Subopercular; SY, Symplectic; TC. S, tectum Synoticum; TC. P, tectum posterius; TM, taenia marginalis; TR, trabecula communis. Abbreviations in italic show unstained bony structures. 


\section{DAY 19}

\section{OSTEOCRANIUM (FIG. 12)}

At this stage all bony structures are stained. Below the anterior extremity of the parasphenoid has appeared a small vomer, elongated in shape and ending with a protuberance. The bony Splanchnocranium now possesses thin palatines which have appeared in front of the anterior tips of the ectopterygoids. Lastly, the ceratohyal, interhyal, and urohyal ossifications have appeared.

FIG. 12. Scophthalmus maximus:

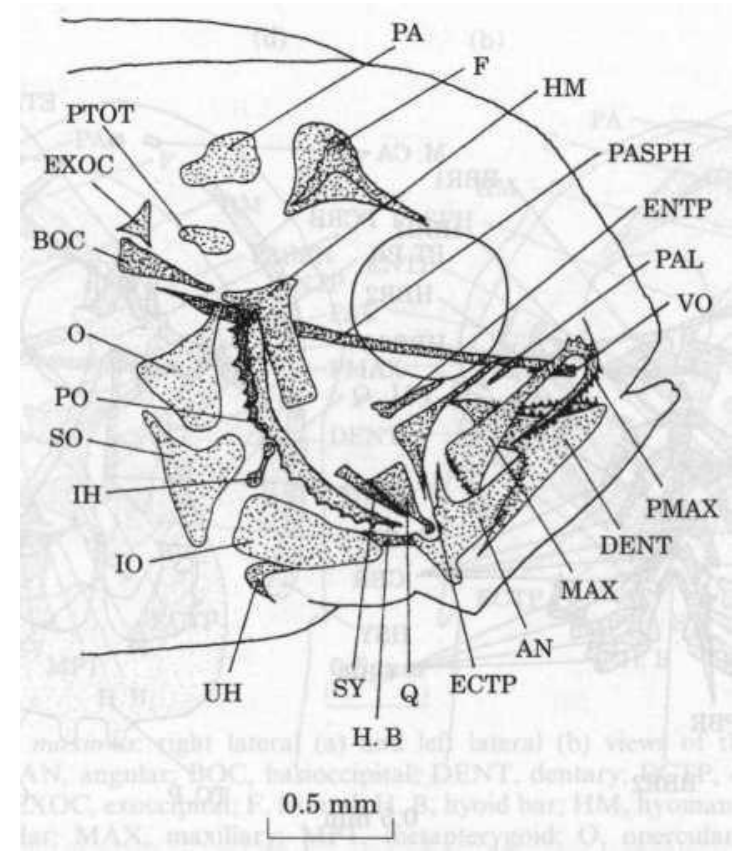

lateral view of the osteocranium of a 19-day-old fry; AN, angular; BOC, basioccipital; DENT, dentary; ECTP, ectopterygoid; ENTP, entopterygoid; EXOC, exoccipital; F, frontal; H. B, hyoid bar; HM, hyomandibular; $\mathrm{IH}_{5}$ interhyal; IO, Interopercular; MAX, maxillary; O, opercular; PA, parietal; PAL, palatine; PASPH, parasphenoid; PMAX, premaxillary; PO, preopercular; PTOT, pterotic; Q, quadrate; SO, Subopercular; SY, Symplectic; UH, urohyal; VO, vomer.

\section{DAY 20}

\section{CHONDROCRANIUM [FIG. 13(A), (B) AND (C)]}

The chondrocranium and Splanchnocranium do not display any new elements. Regression has reached the hyoid bars. Each has split into two clearly distinct elements. The distal element has the shape of an inverted $\mathrm{Y}$ and the massive proximal element displays several digitations. The fifth Ceratobranchials are regressing centrally.

The dorsal fin has moved forward to almost touch the front of the braincase dome, and the distance between the taenia marginalis and the side of the head has increased. 
FIG. 13. Scophthalmus maximus:

(a)

(b)

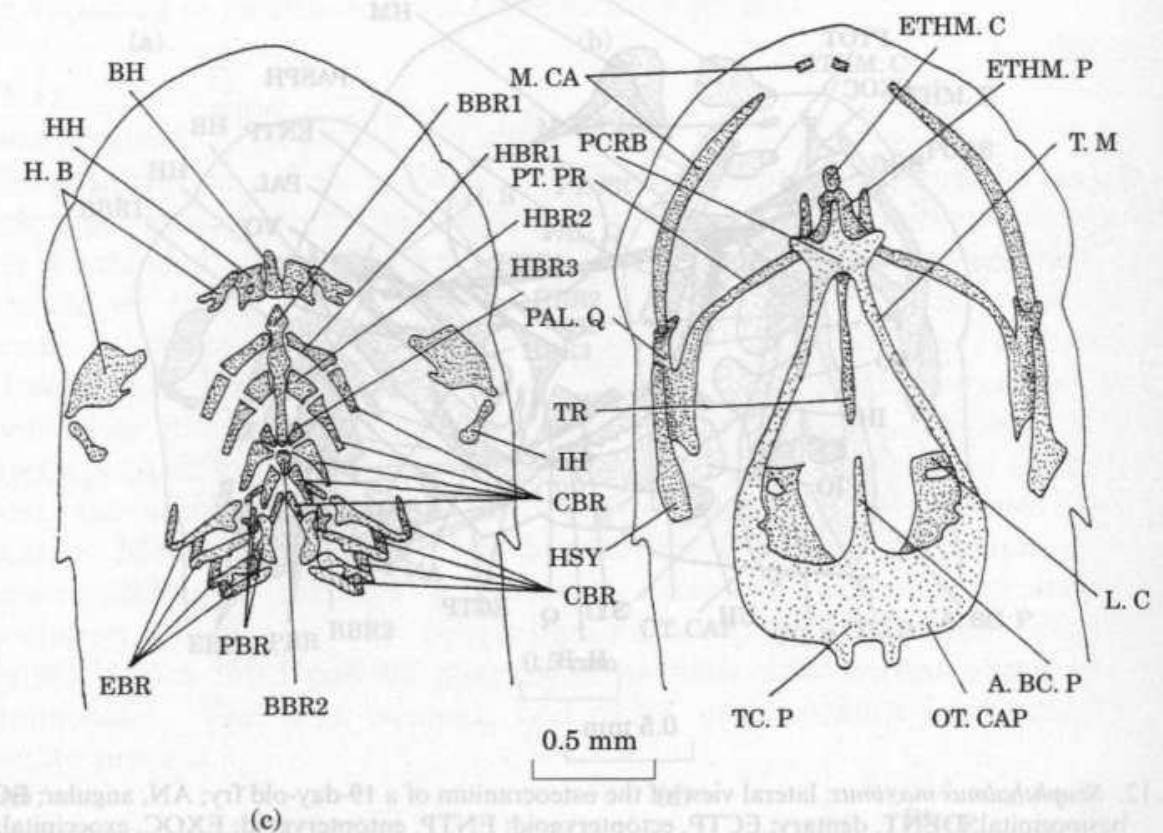

(c)

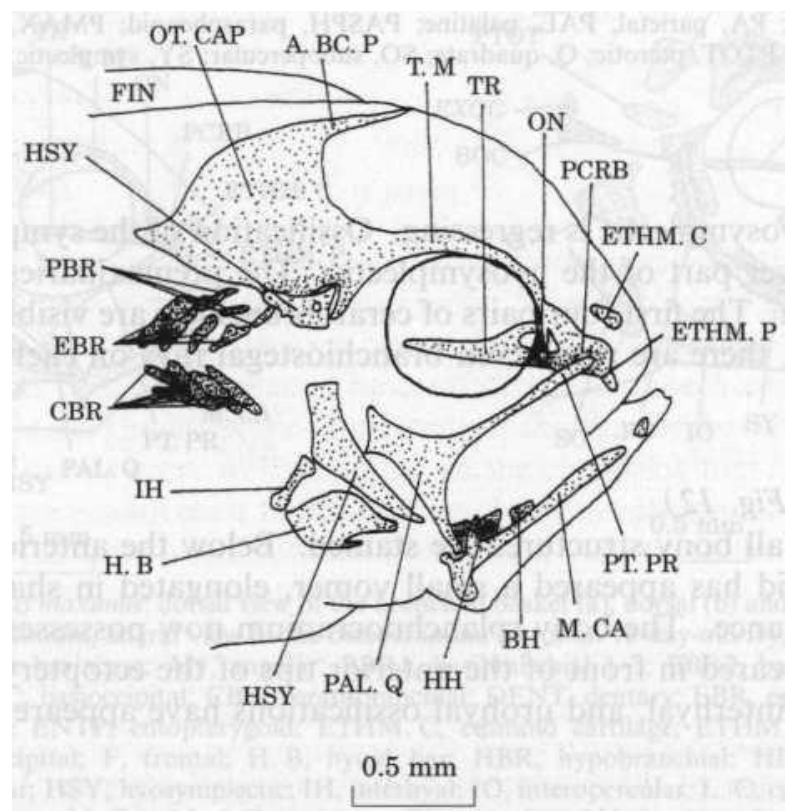

dorsal view of the branchial basket (a), dorsal (b) and lateral (c) views of the chondrocranium of a 20-day-old fry; A. BC. P, anterior process of the braincase; BBR1, basibranchial 1 3; BBR2, basibranchial 4; BH, basihyal; CBR, Ceratobranchial; EBR, epibranchial; ETHM. C, ethmoid cartilage; ETHM. P, ethmoid plate; H. B, hyoid bar; HBR. hypobranchial; HH, hypohyal; HSY, hyosymplectic; IH, interhyal; L. C, commissura lateralis; M. CA, Meckel's cartilage; ON, lamina orbitonasalis; OT. CAP, otic capsule; PAL. Q, palatoquadrate; PBR, pharyngobranchial; PCRB. lamina precerebralis; PT. PR, pterygoid process; TC. P, tectum posterius; TM, taenia marginalis; TR, trabecula communis. 


\section{OSTEOCRANIUM [FIG. 14(A) AND (B)]}

The parasphenoid displays two lateral extensions in front of the hyomandibulars. The metapterygoids are now present in the Splanchnocranium. The entopterygoids almost totally occupy the central parts of the cheeks. Asymmetries have appeared. The right entopterygoid is clearly narrower than the left one, the right ectopterygoid is longer and broader than the left one, the right palatine is longer than the left one, the right quadrate has a much broader ventral edge than does the left quadrate, and the right symplectic is shorter than the left one.

FIG. 14. Scophthalmus maximus:

(a)

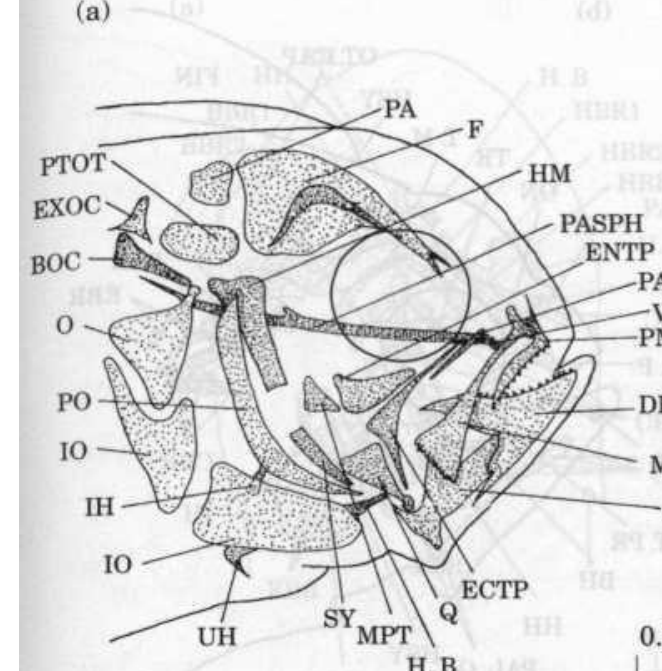

(b)

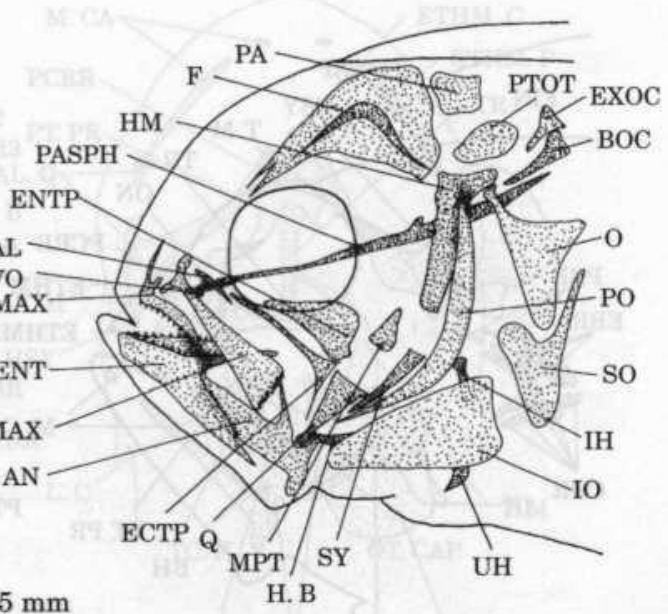

right lateral (a) and left lateral (b) views of the osteocranium of a 20-day-old fry; AN, angular; BOC, basioccipital; DENT, dentary; ECTP, ectopterygoid; ENTP, entopterygoid; EXOC, exoccipital; $\mathrm{F}$, frontal; $\mathrm{H}$. B, hyoid bar; HM, hyomandibular; $\mathrm{IH}$, interhyal; IO, interopercular; MAX, maxillary; MPT, metapterygoid; $\mathrm{O}$, opercular; PA, parietal; PAL, palatine; PASPH, parasphenoid; PMAX, premaxillary; PO, preopercular; PTOT, pterotic; Q, quadrate; SO, subopercular; SY, symplectic; UH, urohyal; VO, vomer.

\section{DAY 23}

\section{CHONDROCRANIUM [FIG. 15(A) AND (B)]}

The anterior part of the braincase dome is finally beginning to regress. The right taenia marginalis previously fused with the lamina precerebralis now separates from it. The ethmoid cartilage continues to develop. The region where each palatoquadrate articulates with the lower jaw has separated from the remainder of the palatoquadrate and is reduced to a cartilaginous stump. One digitation of each hyoid bar produces a long cartilaginous bar extending to the hypohyal.

FIG. 15. Scophthalmus maximus: 


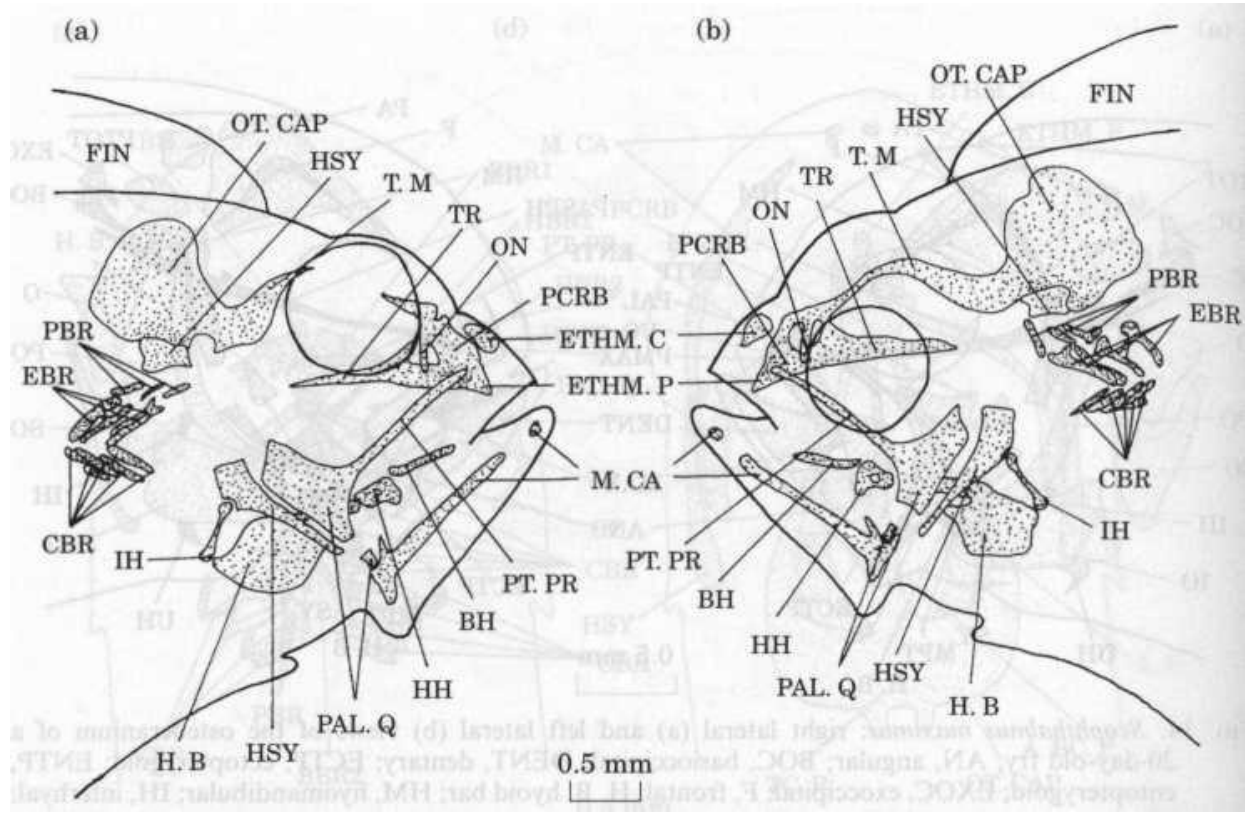

right lateral (a) and left lateral (b) views of the chondrocranium of a 23-day-old fry; BH, basihyal; CBR, Ceratobranchial; EBR, epibranchial; ETHM. C, ethmoid cartilage; ETHM. P, ethmoid plate; H. B, hyoid bar; HBR, hypobranchial; HH, hypohyal; HSY, hyosymplectic; IH, interhyal; M. CA, Meckel's cartilage; ON, lamina orbitonasalis; OT. CAP, otic capsule; PAL. Q, palatoquadrate; PBR, pharyngobranchial; PCRB, lamina precerebralis; PT. PR, pterygoid process; TM, taenia marginalis; TR, trabecula communis.

\section{DAY 25}

\section{CHONDROCRANIUM [FIG. 16(A)-(D)]}

The right taenia marginalis curves towards the left side of the head. The upper part of each hyomandibular is reduced. The fourth epibranchials each have an uncinate process that abuts the process of each third epibranchial. In this way, the third and fourth epibranchials are in contact and together take the general shape of an $\mathrm{H}$. The dorsal fin continues its progression, reaching the front of the otic capsule.

\section{OSTEOCRANIUM [FIG. 17(A), (B) AND (C)]}

In the ethmoid region, along the anterior tips of the frontals, several bony elements now appear: a pair of flat nasals with a small hole through each, a pair of lateral ethmoids just behind the nasals, and a mesethmoid implanted between the nasals. The otic region now displays sphenotics and prootics. The orbitotemporal region is enriched with a pair of pleurosphenoids limiting the posterior walls of the orbits. To the rear of the neurocranium, a Supraoccipital and a pair of epiotics close the cranial vault dorso-posteriorly. The vomer is fused with the anterior end of the parasphenoid. All elements of the hyobranchial system are ossified, except for the anterior extremities of the basihyal and second basibranchial, the extremities of the Ceratobranchials, epibranchials, pharyngobranchials, and the regions where the hypobranchials articulate with the basihyal. Two ossification sites have appeared at the level of the hyoid bar, giving rise to a short epihyal and, in front of the ceratohyals, two hypohyals. The 

is stuck against the left frontal and pushes it leftward so that the two frontals form a convex curvature in the direction of the zenithal face. Two new asymmetries are conspicuous in the Splanchnocranium: the right mandible is somewhat longer than the left one, this difference being due to the difference in length between the two angulars. Also, the retroarticular process of the right angular is more robust than that of the left retroarticular. The branchial basket, on the other hand, displays no asymmetry.

FIG. 16. Scoplithalmus maximus:

(a)
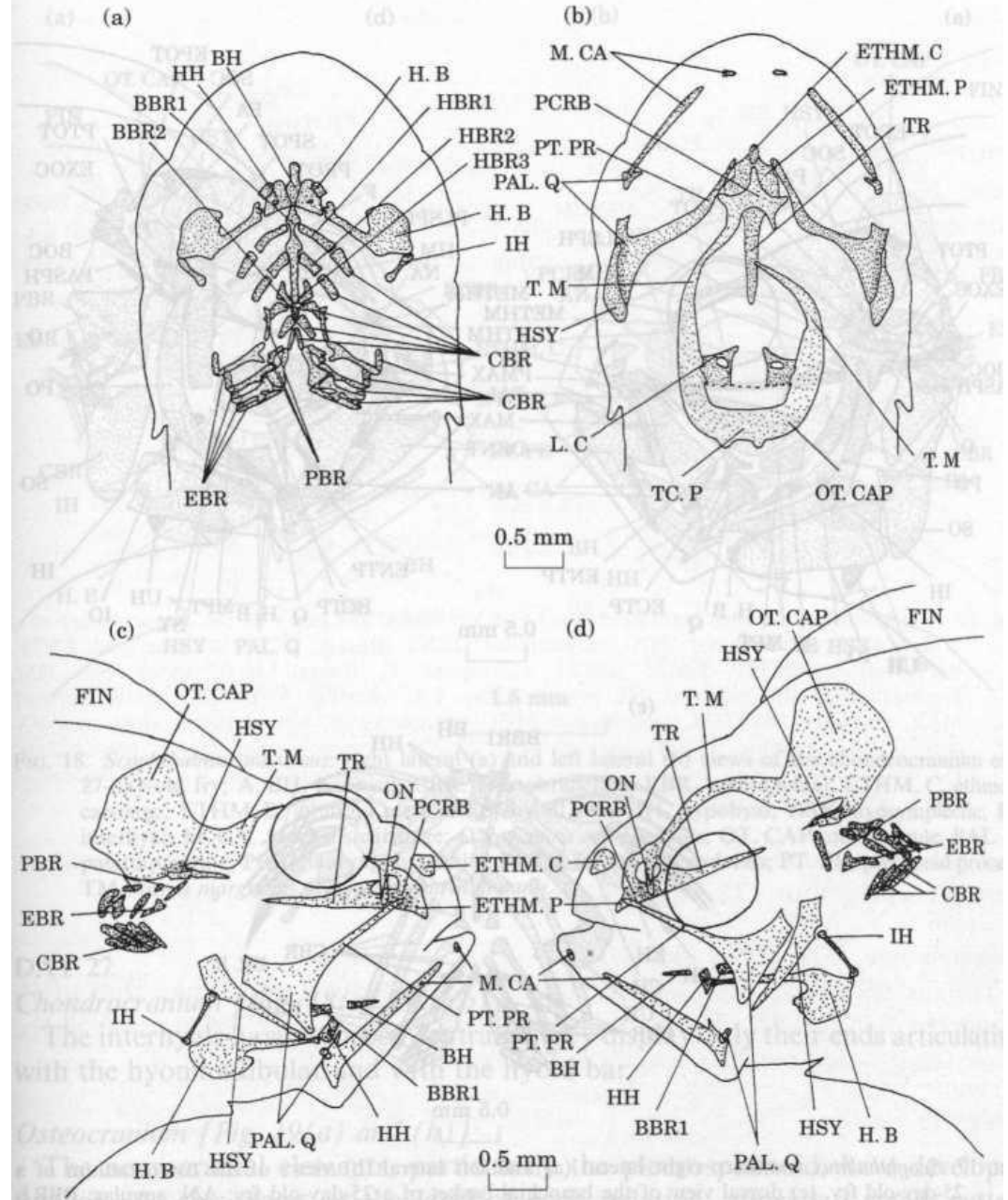

dorsal view of the branchial basket (a), dorsal (b) right lateral (c) and left lateral (d) views of the chondrocranium of a 25day-old fry; (b) The dotted lines indicate regions where cartilaginous elements have regressed; BBR1, basibranchial 1-3; BBR2, basibranchial 4; BH, basihyal; CBR, Ceratobranchial; EBR, epibranchial; ETHM. C, ethmoid cartilage; ETHM. P, ethmoid plate; H. B, hyoid bar; HBR, hypobranchial; HH, hypohyal; HSY, hyosymplectic; IH, interhyal; L. C, commissura lateralis: M. CA, Meckel's cartilage; ON, lamina orbitonasalis: OT. CAP, otic capsule; PAL. Q, palatoquadrate; PBR, pharyngobranchial; PCRB, lamina precerebralis: PT. PR, pterygoid process; TC. P, tectum posterius: TM, taenia marginalis: TR. trabecula communis. 
FIG. 17. Scophthalmus maximus:

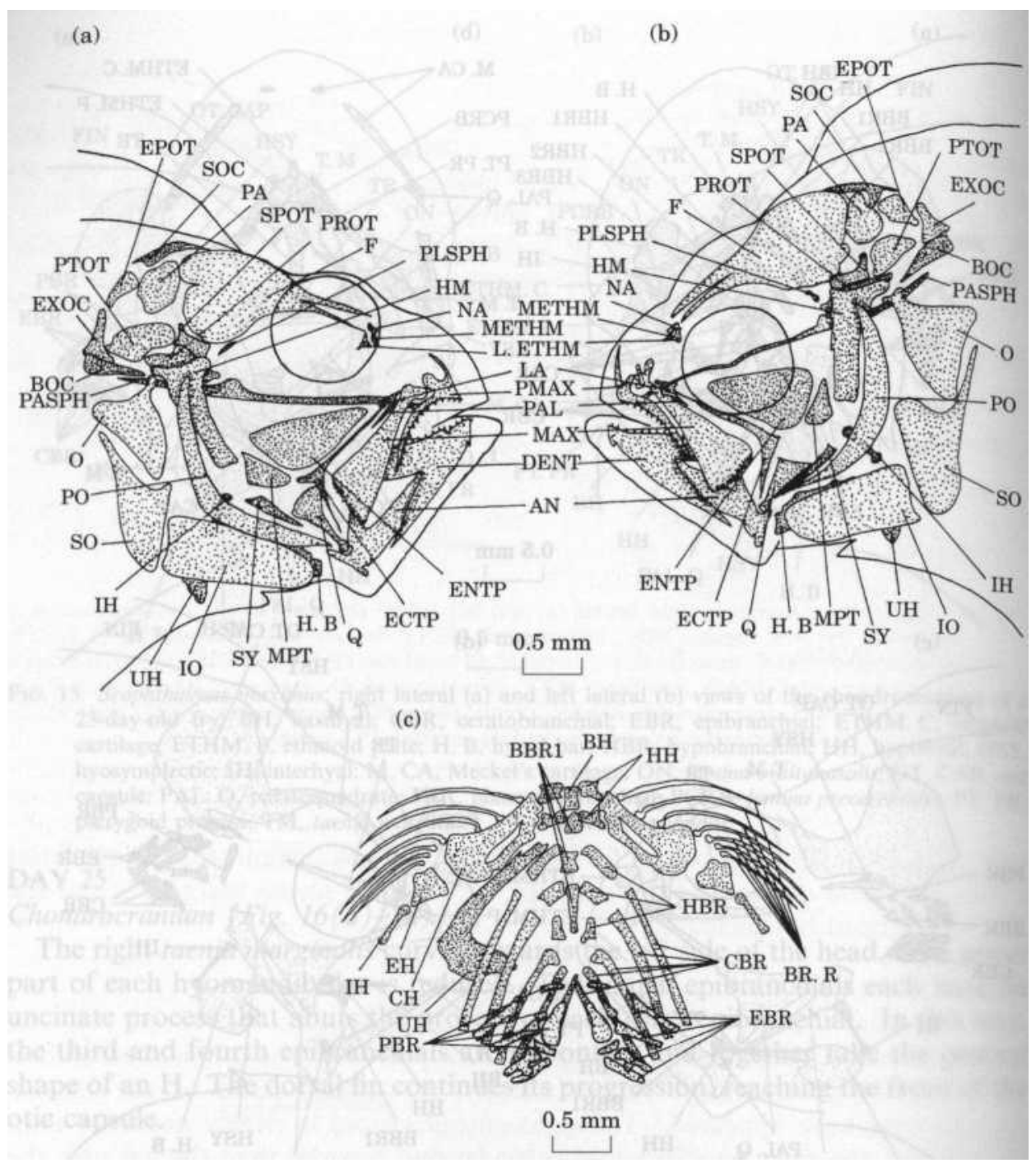

right lateral (a) and left lateral (b) views of the osteocranium of a 25-day-old fry, (c) dorsal view of the branchial basket of a 25-day-old fry; AN, angular; BBR1, basibranchial 1-3; $\mathrm{BH}$, basihyal; $\mathrm{BOC}$. basioccipital; $\mathrm{BR}$. $\mathrm{R}$, branchiostegal rays; CBR. Ceratobranchial; CH, ceratohyal; DENT, dentary; EBR, epibranchial; ECTP. ectopterygoid; EH, epihyal; ENTP, entopterygoid; EPOT, epiotic; EXOC, exoccipital; $F$, frontal; $H$. $B$, hyoid bar; HBR, hypobranchial; HH, hypohyal; HM, hyomandibular; IH, interhyal; IO, interopercular; LA, lacrymal; L. ETHM, lateral ethmoid; MAX, maxillary; METHM, mesethmoid; MPT, metapterygoid; NA, nasal; O, opercular; PA. parietal; PAL, palatine; PASPH, parasphenoid: PBR. pharyngobranchial; PLSPH, pleurosphenoid; PMAX, premaxillary; PO, preopercular; PROT. prootic; PTOT, pterotic; Q, quadrate; SO, Subopercular; SOC, Supraoccipital; SPOT, sphenotic; SY, symplectic; UH, urohyal.

\section{DAY 27}

\section{CHONDROCRANIUM [FIG. 18(A) AND (B)]}

The interhyals have resorbed centrally; they display only their ends articulating with the hyomandibular and with the hyoid bar. 
FIG. 18. Scophthalmus maximus:

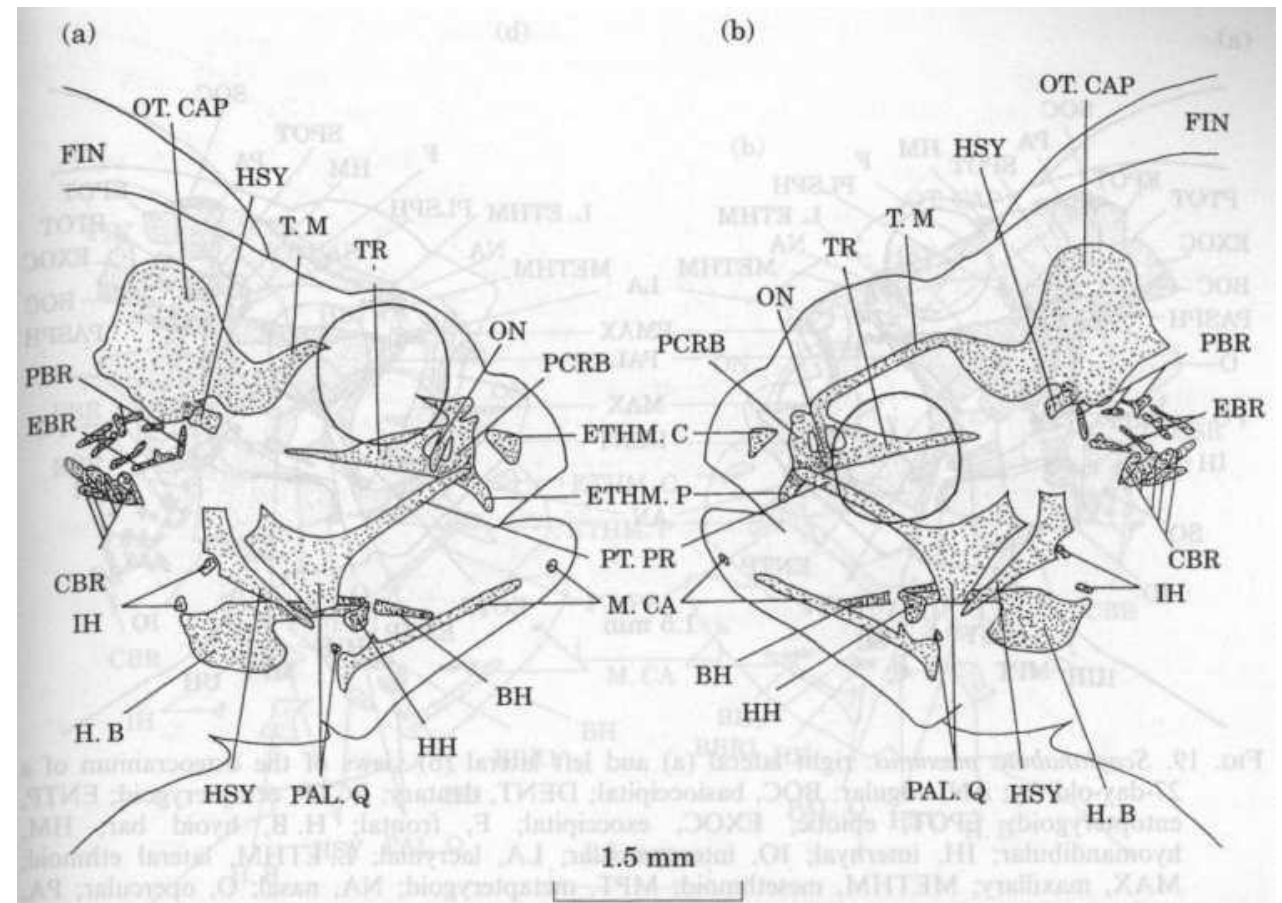

right lateral (a) and left lateral (b) views of the chondrocranium of a 27-day-old fry; A. BH, basihyal; CBR, Ceratobranchial; EBR, epibranchial; ETHM. C, ethmoid cartilage; ETHM. P, ethmoid plate; H. B, hyoid bar; HH, hypohyal; HSY, hyosymplectic; IH, interhyal; M. CA, Meckel's cartilage; ON, lamina orbitonasalis: OT. CAP, otic capsule; PAL. Q, palatoquadrate; PBR. pharyngobranchial; PCRB. lamina precerebralis: PT. PR. pterygoid process; TM, taenia marginalis: TR, trabecula communis.

\section{OSTEOCRANIUM [FIG. 19(A) AND (B)]}

The neurocranial elements, particularly the pleurosphenoid, have developed further. At this stage, at the level of each palatine, appears a small, gutter-shaped lacrymal with a hole through it. The nasals are transformed and have become upward-curving gutters. The asymmetry affects the lateral ethmoids and lacrymals. The latter show differences in shape and position: the right lacrymal has a more star-shaped contour than its homologue on the left and it occupies a more dorsal position. The triangular lateral ethmoids differ in size and shape, the right one being larger than the left one. 
FIG. 19. Scophthalmus maximus:

(a)

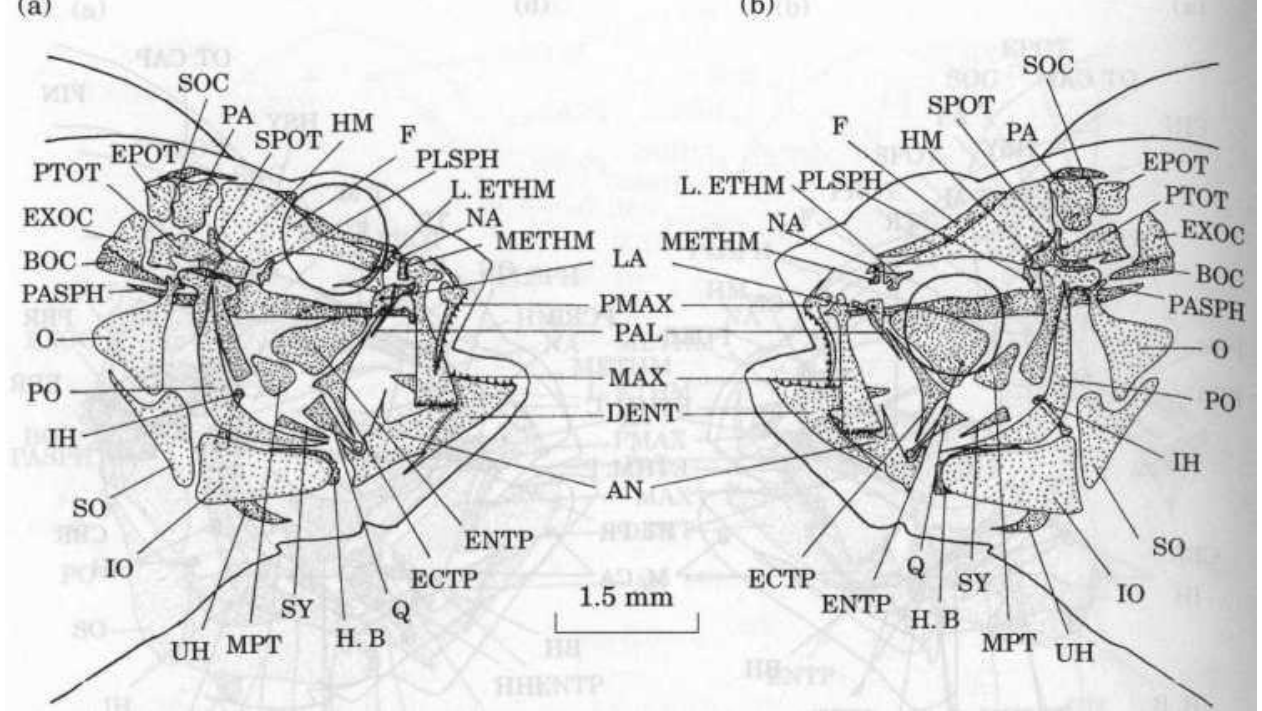

right lateral (a) and left lateral (b) views of the OSteocranium of a 27-day-old fry; AN, angular; BOC, basioccipital; DENT, dentary; ECTP, ectopterygoid; ENTP, entopterygoid; EPOT, epiotic; EXOC, exoccipital; F, frontal; H. B, hyoid bar; HM, hyomandibular; IH, interhyal; IO, interopercular; LA, lacrymal; L. ETHM, lateral ethmoid; MAX, maxillary; METHM, mesethmoid; MPT, metapterygoid; NA, nasal; O, opercular; PA, parietal; PAL, palatine; PASPH, parasphenoid; PLSPH, pleurosphenoid; PMAX, premaxillary; PO, preopercular; PTOT, pterotic; Q, quadrate; S, skull; SO, Subopercular; SOC, Supraoccipital; SPOT, sphenotic; SY, Symplectic; UH, urohyal.

\section{DAY 29}

\section{CHONDROCRANIUM [FIG. 20(A) AND (B)]}

The otic capsule is resorbed impressively, becoming isolated from the taeniae marginales. The left taenia marginalis is separating from the lamina precerebralis. The branchial basket appears dissimilar: only the extremities of the cerato-, epi-, and Pharyngobranchials, a scattered basibranchial 1 , and a reduced basihyal remain cartilaginous. The hyosymplectic is splitting into two parts.

\section{OSTEOCRANIUM [FIG. 20(C) AND (D)]}

The surface of the right lateral ethmoid has increased more than that of its counterpart on the left. The right lacrymal retains its more or less star-like shape while the left lacrymal has lengthened dorso-ventrally. 
FIG. 20. Scophthalmus maximus:

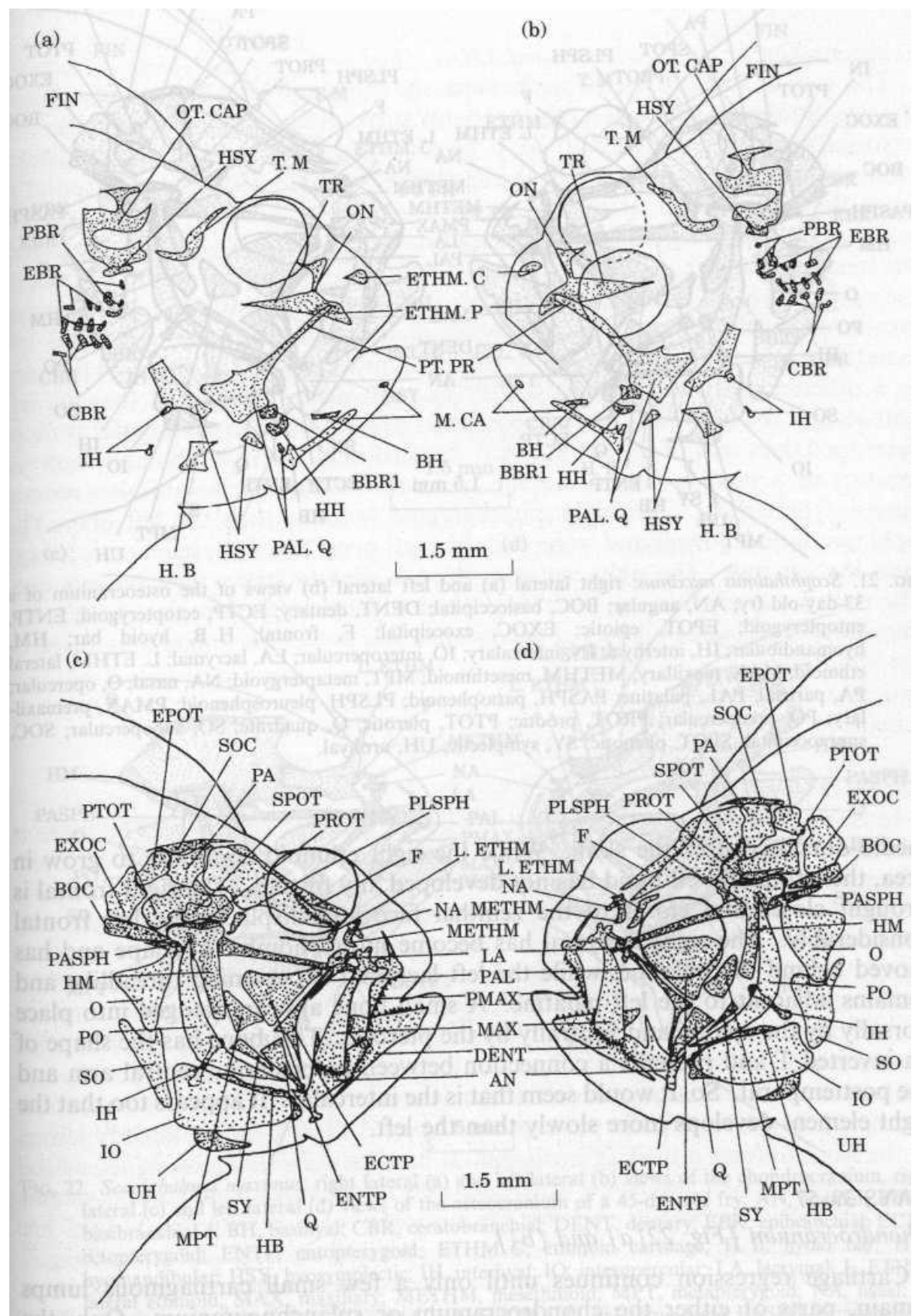

right lateral (a) and left lateral (b) views of the chondrocranium, right lateral (c) and left lateral (d) views of the osteocranium of a 29-day-old fry; AN, angular; BBR1 basibranchial 1-3; BH, basihyal; BOC, basioccipital; CBR, Ceratobranchial; DENT, dentary; EBR. epibranchial; ECTP, ectopterygoid; ENTP, entopterygoid; EPOT, epiotic; ETHM. C, ethmoid cartilage; ETHM. P, ethmoid plate; EXOC, exoccipital; F, frontal; H. B, hyoid bar; HH, hypohyal; HM, hyomandibular; HSY, hyosymplectic; IH, interhyal; IO, interopercular; LA, lacrymal; L. ETHM, lateral ethmoid; MAX, maxillary; M. CA, Meckel's cartilage; METHM, mesethmoid; MPT, metapterygoid; NA, nasal; O, opercular; ON, lamina orbitonasalis; OT. CAP, otic capsule; PA, parietal; PAL, palatine; PAL. Q, palatoquadrate; PASPH, parasphenoid; PBR. Pharyngobranchial; PLSPH, pleurosphenoid; PMAX, premaxillary; PO, preopercular; PROT. prootic; PTOT, pterotic; PT. PR, pterygoid process; Q, quadrate; SO, Subopercular; SOC. Supraoccipital; SPOT, sphenotic; SY, Symplectic; TM. taenia marginalis; TR, trabecula communis; $\mathrm{UH}$, urohyal. 


\section{DAYS 33->45}

\section{OSTEOCRANIUM [FIG. 21(A) AND (B)]}

The anterior region of the neurocranium is undergoing profound modifications in relation to the migration of the right eye. The latter has reached the medio-dorsal crest of the skull. While the right ethmoid continues to grow in area, the left lateral ethmoid has not developed any further. The right frontal is brought closer and closer to the zenithal face and displaces the left frontal considerably. The right lacrymal has become more rhombic in shape and has moved behind the palatine, while the left lacrymal is becoming threadlike and remains adjacent to the left palatine. A small bone appears wedged into place dorsally by the epiotic and ventrally by the pterotic. This bone has the shape of an inverted $\mathrm{T}$ and presents a connection between its posterior ventral arm and the posttemporal. So, it would seem that is the intercalar. It appears too that the right element develops more slowly than the left.

FIG. 21. Scophthalmus maximus:

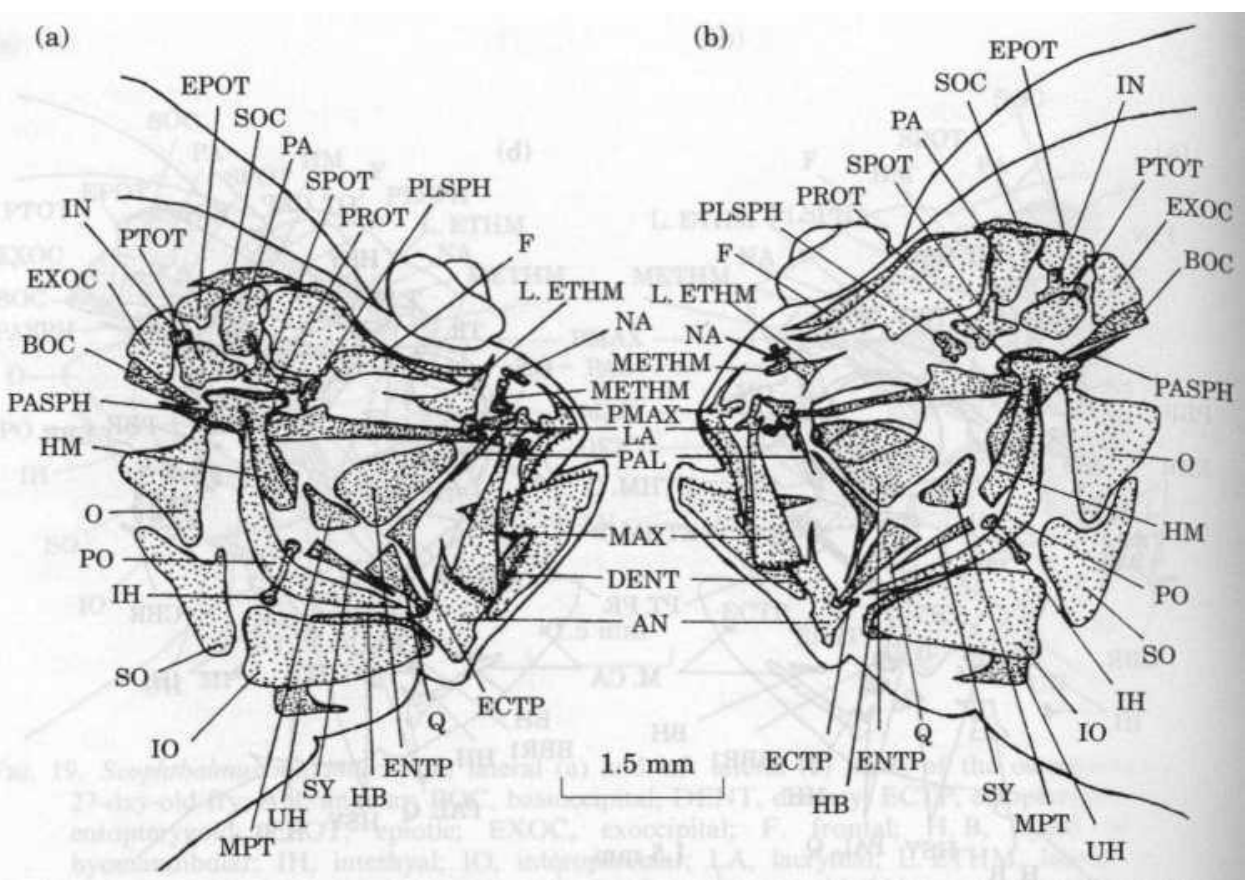

right lateral (a) and left lateral (b) views of the OSteocranium of a 33-day-old fry; AN, angular; BOC, basioccipital; DENT, dentary; ECTP, ectopterygoid; ENTP, entopterygoid; EPOT, epiotic; EXOC, exoccipital; F, frontal; H. B, hyoid bar; HM, hyomandibular; IH, interhyal; IN, intercalary; IO, interopercular; LA, lacrymal; L. ETHM, lateral ethmoid; MAX, maxillary; METHM, mesethmoid; MPT, metaptergyoid; NA, nasal; O, opercular; PA, parietal; PAL, palatine; PASPH, parasphenoid; PLSPH, pleurosphenoid; PMAX, premaxillary; PO, preopercular; PROT, prootic; PTOT, pterotic; Q, quadrate; SO, Subopercular; SOC, Supraoccipital; SPOT, phenotic; SY, symplectic; UH, urohyal.DAYS 39-57

\section{CHONDROCRANIUM [FIG. 22(A) AND (B)]}

Cartilage regression continues until only a few small cartilaginous lumps remain, parts of either the chondrocranium or Splanchnocranium. Only the ethmoid cartilage shows no regression. At 39 days, the dorsal fin reaches the 
back of the right orbit and progresses until the right eye has passed completely over to the left side after 45 days. In the following days, the right eye moves closer to the left one. Metamorphosis is considered complete at around 60 days.

\section{OSTEOCRANIUM [FIGS 22(C), (D) AND 23(A), (B)]}

The vomer, stuck to the parasphenoid, shows lateral wing-like extensions and the nasals take the shape of a tube probably surrounding the sensory canals. The asymmetries noted on day 20 have become more marked. The right lateral ethmoid has moved to the top of the head and has fused with the right frontal to form the nadiral wall of the migrating eye's orbit. The left lateral ethmoid retains its initial position. The median crest of the mesethmoid is tipped towards the blind side and that of the vomer, not very well developed, is tipped towards the oculate side. The right lacrymal has developed little while the left lacrymal grows larger and larger. The difference in length between the right and left mandibles is no longer due solely to the different lengths of the angulars but also to a difference between the dentaries: the right dentary is longer than its left homologue. Furthermore, the retroarticular process of the right angular is more developed than that of the left angular. Nevertheless, the mandibular symphysis remains in a median plane and the quadrato-mandibular joints also remain almost symmetrical. The right entopterygoid is lower than the left one. The right quadrate is longer and wider than the left one. The right palatine is longer than the left one. The right metapterygoid is slightly larger than the left one. The right ectopterygoid is a little longer and wider than the left one. Lastly, the maxillaries and premaxillaries of the occulate side are a little longer than those of the blind side. 


\section{FIG. 22. Scophthalmus maximus:}

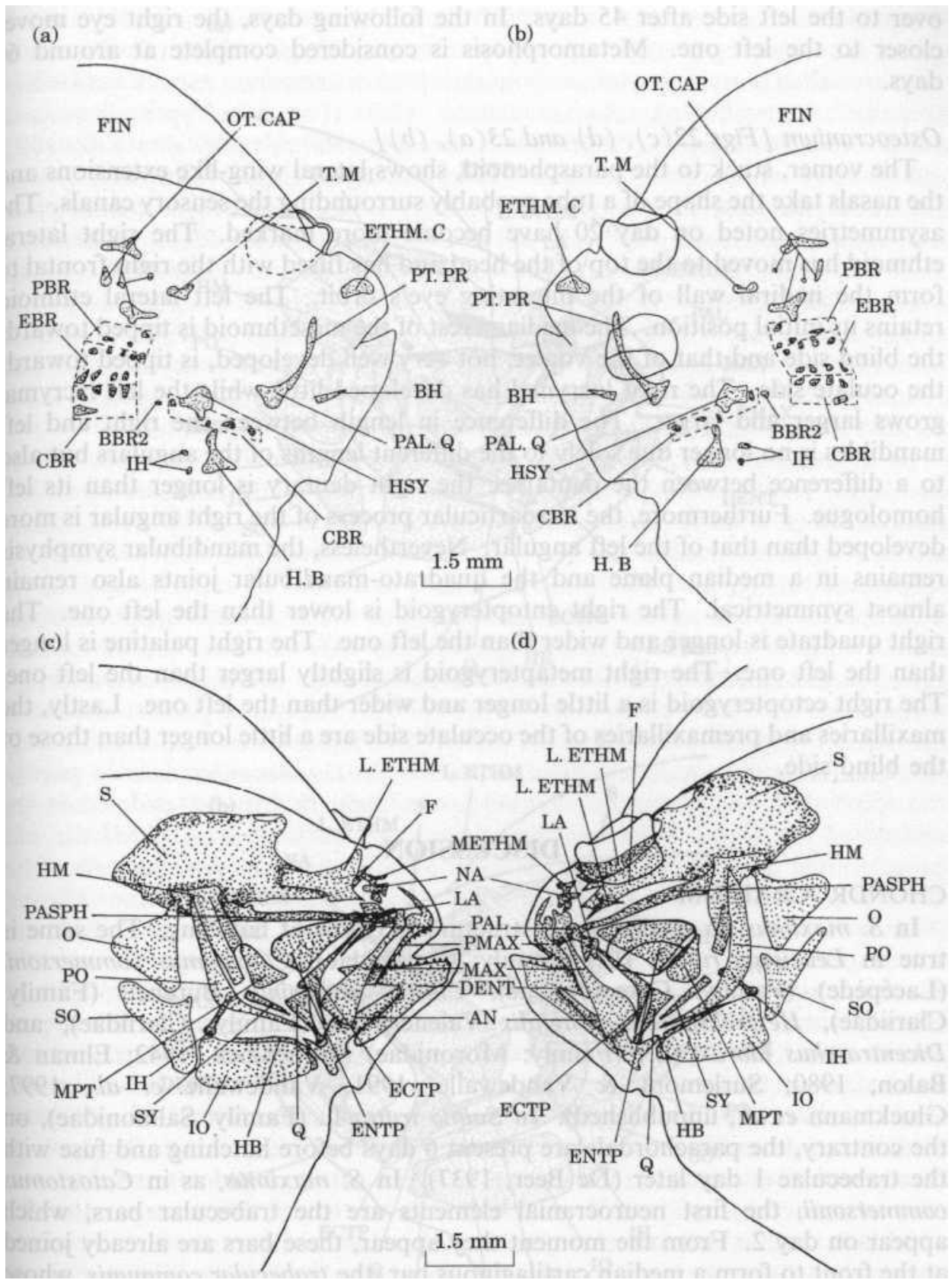

right lateral (a) and left lateral (b) views of the chondrocranium, right lateral (c) and left lateral (d) views of the osteocranium of a 45-day-old fry; AN, angular; BBR2, basibranchial 4; BH, basihyal; CBR, Ceratobranchial; DENT, dentary; EBR, epibranchial; ECTP, ectopterygoid; ENTP, entopterygoid; ETHM. C, ethmoid cartilage; H. B, hyoid bar; HM, hyomandibular; HSY, hyosymplectic; IH, interhyal; IO, interopercular; LA, lacrymal; L. ETHM, lateral ethmoid; MAX, maxillary; METHM, mesethmoid; MPT, metapterygoid; NA, nasal; O, opercular; OT. CAP, otic capsule; PAL, palatine; PAL. Q. palatoquadrate; PASPH, parasphenoid; PBR, Pharyngobranchial; PMAX, premaxillary; PO, preopercular; PT. PR. pterygoid process; Q, quadrate; S, skull; SO, Subopercular; SY, Symplectic; TM, taenia marginalis: UH, urohyal. 
FIG. 23. Scophthalmus maximus:

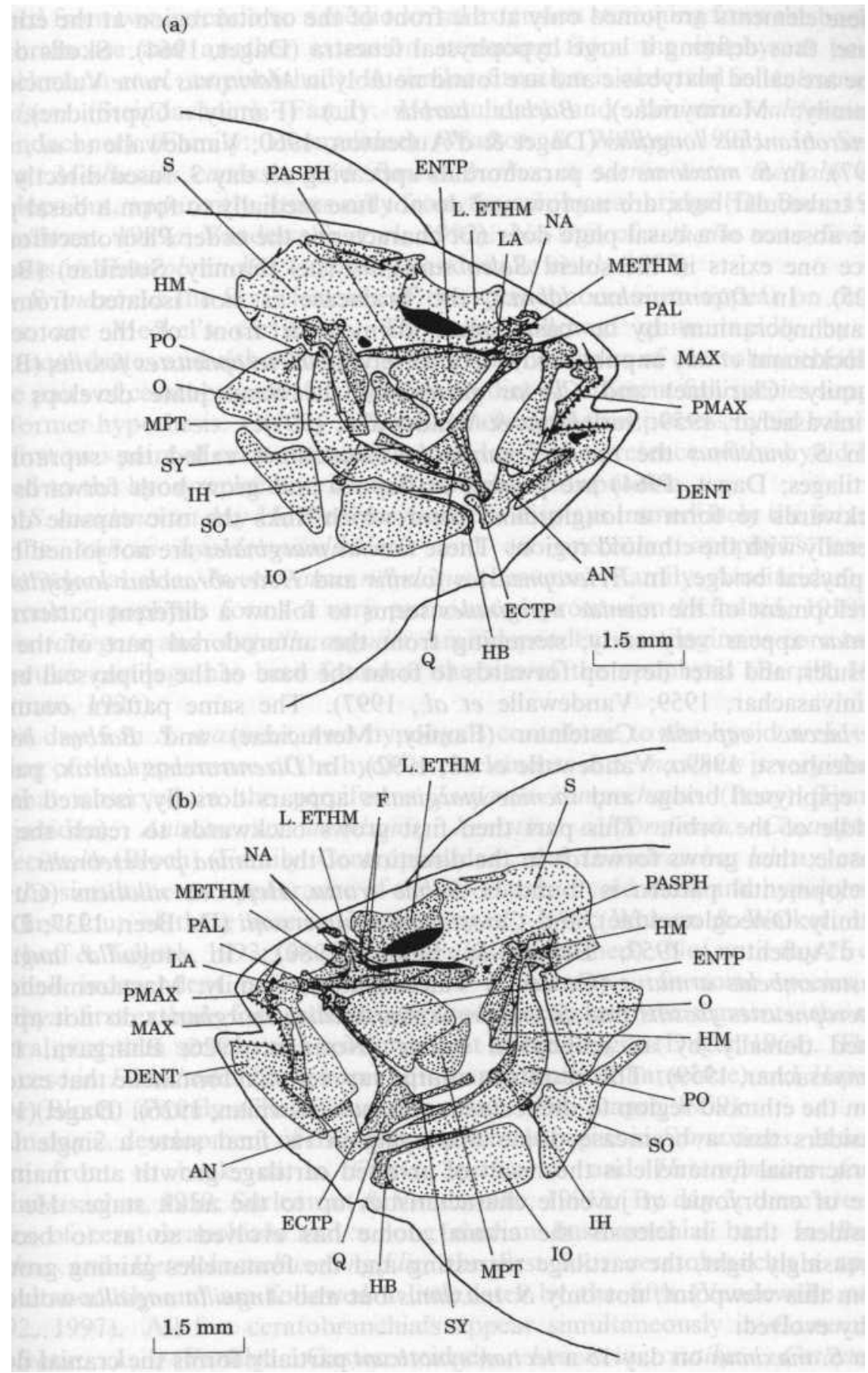

right lateral (a) and left lateral (b) views of the osteocranium of a 57-day-old fry; AN, angular; DENT, dentary; ECTP, ectopterygoid; ENTP, entopterygoid; H. B, hyoid bar; HM, hyomandibular; IH, interhyal; IO, interopercular; LA, lacrymal; L. ETHM, lateral ethmoid; MAX, maxillary; METHM, mesethmoid; MPT, metapterygoid; NA, nasal; O, opercular; PAL, palatine; PASPH, parasphenoid; PMAX, premaxillary; PO, preopercular; Q, quadrate; S, skull; SO, subopercular; SY, symplectic; UH, urohyal. 


\section{DISCUSSION}

\section{CHONDROCRANIUM}

In S. maximus no cartilaginous structure is visible at hatching. The same is true in Leuciscus rutilus (L.) (Family: Cyprinidae), Catostomus Commersonii (Lacépède) (Family: Catostomidae), Clarias gariepinus Burchell (Family: Clariidae), Heterobranchus longifilis Valenciennes (Family: Clariidae), and Dicentrarchus labrax (L.) (Family: Moronidae) (Hubendick, 1942; Elman \& Balon, 1980; Surlemont \& Vandewalle, 1991; Vandewalle et al., 1997; Gluckmann et al., unpublished). In Salmo trutta L. (Family: Salmonidae), on the contrary, the parachordals are present 6 days before hatching and fuse with the trabeculae 1 day later (De Beer, 1937). In S. maximus, as in Catostomus Commersonii, the first neurocranial elements are the trabecular bars, which appear on day 2. From the moment they appear, these bars are already joined at the front to form a median cartilaginous bar, the trabecular communis, whose rostral extremity widens out a little later into an ethmoid plate. Skulls of this type are called tropibasic and are characteristic of fish with heads that are higher than they are wide, often with large eyes and close ocular lobes (Daget, 1964). Leuciscus rutilus, Dicentrarchus labrax, and Anguilla anguilla (L.) (Family: Anguillidae) present this type of skull (Norman, 1926; Hubendick. 1942; Gluckmann et al., unpublished). However, when the skull is quite flat and the eyes of the fish are small, the anterior parts of the trabeculae remain apart.

These elements are joined only at the front of the orbital region at the ethmoid plate, thus defining a large hypophyseal fenestra (Daget, 1964). Skulls of this type are called platybasic and are found notably in Mormyrus rume Valenciennes (Family: Mormyridae), Barbus barbus (L.) (Family: Cyprinidae), and Heterobranchus longifilis (Daget \& d'Aubenton, 1960; Vandewalle et al., 1992, 1997). In S. maximus the parachordals appearing on day 3, fused directly with the trabecular bars, are narrow and do not fuse medially to form a basal plate. The absence of a basal plate does not characterize the order Pleuronectiformes, since one exists in the soleid Solea variegata (L.) (Family: Soleidae) (Berrill, 1925). In Dicentrarchus labrax, the braincase is not isolated from the Splanchnocranium by a basal plate formed in front of the notochord (Gluckmann et al., unpublished). Conversely, in Heteropneustes fossilis (Bloch) (Family: Clariidae) and Clarias gariepinus, the basal plate develops early (Srinivasachar, 1959; Surlemont \& Vandewalle, 1991).

In $S$. maximus the taeniae marginales (sometimes called the supraorbital cartilages; Daget, 1964) are present at day 10 and grow both forwards and backwards to form a longitudinal band which links the otic capsule dorsolaterally with the ethmoid region. These taeniae marginales are not joined by an epiphyseal bridge. In Heteropneustes fossilis and Heterobranchus longifilis, the development of the taeniae marginales seems to follow a different pattern: the taeniae appear very early, stemming from the anterodorsal part of the otic capsules, and later develop forwards to form the base of the epiphyseal bridge 
(Srinivasachar, 1959; Vandewalle et al., 1997). The same pattern occurs in Merluccius capensis Castelnau (Family: Merlucidae) and Barbus barbus (Badenhorst, 1989a; Vandewalle et al., 1992). In Dicentrarchus labrax, part of the epiphyseal bridge and taeniae marginales appears dorsally, isolated in the middle of the orbit. This part then first grows backwards to reach the otic capsule, then grows forwards in the direction of the lamina precerebralis. This developmental pattern is found in Salmo trutta, Heterotis niloticus (Cuvier) (Family: Osteoglosseidae), and Catostomus Commersonii (De Beer, 1937; Daget \& d'Aubenton, 1957; Elman \& Balon, 1980). In Anguilla anguilla, Mastacembelus armatus (Cuvier \& Valenciennes) (Family: Mastacembelidae), Heteropneustes fossilis and S. maximus, the taeniae marginales do not appear joined dorsally by an epiphyseal bridge (Norman, 1926; Bhargava, 1958; Srinivasachar, 1959). This results in a large supracranial fontanelle that extends from the ethmoid region to the tectum synoticum (Norman, 1926). Daget (1964) considers that a braincase dome displaying in its final state a single large supracranial fontanelle is the result of arrested cartilage growth and maintenance of embryonic or juvenile characteristics up to the adult stage. He also considers that in teleosts the cranial dome has evolved so as to become increasingly light, the cartilage regressing and the fontanelles gaining ground. From this viewpoint, not only $S$. maximus but also Anguilla anguilla would be highy evolved.

In S. maximus on day 15 a tectum synoticum partially forms the cranial dome and is extended anteriorly by an anterior mediodorsal process. This anterior process or taenia tecta medialis partially divides the fontanelle lengthwise. The tectum posterius appears a little later. In D. labrax the tectum synoticum appears in the braincase dome after the tectum posterius, and a taenia tecta medialis is formed from two extensions: a mediodorsal extension stemming from the back of the braincase and another extension stemming from the epiphyseal bridge (Gluckmann et al., unpublished). A similar situation is observed in Amsotremus davidsoni (Steindachner) (Family: Haemulidae) and Xenistius Californiensis (Steindachner) (Family: Haemulidae) (Watson \& Walker, 1992). In Salmo trutta, Merluccius capensis, and Barbus barbus, a taenia tecta medialis also develops but, apparently, stems only from the epiphyseal bridge (De Beer, 1937; Badenhorst, 1989a; Vandewalle et al., 1992). No sign of a taenia tecta medialis appears in Heterobranchus longifilis (Vandewalle et al., 1997).

In S. maximus the first elements of the Splanchnocranium appear on day 1. These are Meckel's cartilages. They are followed quite rapidly by the palatoquadrates and either the hyoid bars and two pairs of Ceratobranehials, or three pairs of Ceratobranehials. Previous studies on different fish species support the former hypothesis. On day 2, S. maximus would thus possess hyoid bars and the first two pairs of Ceratobranehials. By day 3, the presence of the hyoid bars linked to the hyosymplectics via an interhyal is indisputable.

In $S$. maximus on day 15 , the hyosymplectic displays immediately the foramen of the truncus hyoideomandibularis and an opercular apophysis on its posterodorsal side. In Ameiurus nebulosus (Lesueur) (Family: Ameiuridae), the opercular apophysis forms a very pronounced protrusion (Kindred, 1919); in 
Solea variegata and Anguilla anguilla, an independent cartilaginous core called opercular cartilage has been found at the site of the apophysis (Berrill, 1925; Norman, 1926).

On day 5 in $S$. maximus, two hypohyals contribute to the hyoid arch. The timing of the appearance of the hyoid arch elements in S. maximus is very similar to that observed in the perciforms Lutjanus campechanus (Poey) (Family: Lutjanidae), Anisotremus davidsonii, Xenistius Californiensis, Centropomus undecimalis (Bloch) (Family: Centropomidae), and Dicentrarchus labrax, except for the simultaneous appearance of the interhyals, hyoid bars, and hypohyals in the first four of these species (Potthoff et al., 1988; Watson \& Walker, 1992; Potthoff \& Tellock, 1993; Gluckmann et al., unpublished). Not until day 15 does a small independent basihyal appear in S. maximus. In some species, the basihyal first extends forwards, then splits into two or three segments, the most rostral part then serving as a reinforcement of the tongue (Daget, 1964). This is the case in Distichodus brevipinnus Günther (Family: Characidae) and Hepsetus odoe (Bloch) (Family: Characidae) (Bertmar, 1959; Daget, 1959).

On day 2, development of the Ceratobranchials begins in $S$. maximus. It occurs from front to back, as in Clarias gariepinus and Heteropneutes fossilis (Srinivasachar, 1959; Surlemont \& Vandewalle, 1991). By day 3, there are four pairs of Ceratobranchials and a long median basibranchial bar. In Barbus barbus and Heterobranchus longifilis, the first four Ceratobranchials appear simultaneously and are followed a little later by the fifth (Vandewalle et al., 1992, 1997). All five Ceratobranchials appear simultaneously in Gasterosteus aculeatus L. (Family: Gasterosteidae), Leuciscus rutilus, Catostomus commersonii, Anisotremus davidsonii, and Xenistius Californiensis (Swinnerton, 1902; Hubendick, 1942; Elman \& Balon, 1980; Watson \& Walker, 1992). On day 5, S. maximus displays a small posterior basibranchial, and on day 6 , three pairs of hypobranchials. The fifth Ceratobranchial and the four epibranchials appear simultaneously on day 10 . The branchial basket displays on day 12 two pharyngobranchials: one facing epibranchials 3 and 4, and one extending epibranchial 2. The pharyngobranchial of the first arch appears on day 15: it does not contribute to the pharyngeal jaws in adult acanthopterygians. In $S$. maximus, the pharyngobranchials thus appear progressively from rear to front as in Barbus barbus (Vandewalle et al., 1992) and Dicentrarchus labrax (Gluckmann et al., unpublished). In Salmo trutta, the order of appearance of the Ceratobranchials, epibranchials, and pharyngobranchials is almost identical to that observed in $S$. maximus. The only differences are that the fourth epibranchial appears later than the first ones and that there are four pharyngobranchials (DeBeer, 1937).

The cartilaginous parts regress in teleosts as the bony skeleton forms, but this regression follows different courses according to the species (Wells, 1923; De Beer, 1937; Vandewalle et al., 1997). On day 10 in S. maximus, the splitting of Meckel's cartilages isolates two small anterior cartilaginous lumps and this is the first sign of regression. On day 15, cartilage regression continues and reaches 
the trabecula communis. On day 18, the centres of the Ceratobranchials and the hyosymplectics undergo resorption. On day 20, the hyoid bar splits into two elements, a small anterior part and a larger posterior one. The anterior cartilage might possibly be a second hypohyal, but Potthoff et al. (1988); Potthoff \& Tellock (1993); Watson \& Walker (1992) and Gluckmann et al. (unpublished) all show that in perciforms, both hypohyals in adults develop in the same cartilaginous lump, corresponding to the first hypohyal. On day 23 in $S$. maximus, the posterior element of the hyoid bar displays a bar-shaped dorsal extension pointing towards the two hypohyals. This cartilaginous extension stemming secondarily from the hyoid bar, may be the base of a beryciform foramen. In Microspathodon chrysurus (Cope) (Family: Pomacentridae) and Centropomus undecimalis, a beryciform foramen exists in the hyoid cartilage (Potthoff et al., 1987; Potthoff \& Tellock, 1993). McAllister (1968) views as primitive the beryciforms and the few families of perciforms (e.g. Pomacentridae and (entropomidae) that possess this beryciform foramen. At this stage in $S$. maximus, the palatoquadrate is split in two, the tectum synoticum and taenia tecta medialis have disappeared, and the right taenia marginalis separates from its lamina orbitonasalis and moves to the left. This is the only metamorphosislinked asymmetry appearing during Chondrocranial development. Several days later (day 29), the left taenia marginalis separates from the corresponding lamina orbitonasalis. Finally, on day 45, resorption leaves only a few small cartilaginous lumps belonging to the neurocranium and Splanchnocranium.

In Solea variegata and Pleuronectes platessa (L.) (Family: Pleuronectidae), the left taenia marginalis, on what is to become the blind nadiral side, disappears shortly after it forms, although the skull is still perfectly symmetrical (Daget, 1964). The right taenia marginalis, on what is to become the sighted zenithal side, is resorbed later (Williams, 1902; Berrill, 1925). In Anguilla anguilla, the taeniae marginales regress and disappear during metamorphosis (Norman, 1926). This transition phase between the leptocephalic larva stage and elver or juvenile stage characteristic of anguilliforms does not, as in flatfish, entail profound cranial modifications resulting in behavioural changes affecting, for instance, the manner of feeding.

In Dicentrarchus labrax, cartilage regression starts with the posterior parts of the trabecular bars, isolating ventrally and very quickly the anterior and posterior parts of the still incomplete neurocranium (Gluckmann et al., unpublished); a similar situation is observed in Merluccius capensis and Barbus barbus (Badenhorst, 1989; Vandewalle et al., 1992). The first four pairs of Ceratobranchials and the hyoid bars of Dicentrarchus labrax start to regress at the next stage, at the same time as Meckel's cartilages, which split as observed in S. maximus (Gluckmann et al., unpublished). In Heterobranchus longifilis, the first signs of resorption appear when the chondrocranium is almost complete: affected are the central parts of the Ceratobranchials and hyoid bars (Vandewalle et al., 1997). In this species, Meckel's cartilages regress later and somewhat differently from those of $S$. maximus: they split into three parts, but the anterior element occupies a position identical to that of the anteriorly isolated element of the other species. Isolation of a cartilaginous element at the front of the 
mandible seems frequent; this fact casts doubt on the purely dermal origin of the dentary bones. These might have absorbed the small anterior cartilaginous elements and thus have a dual origin like the palatines which consist of an auto- and a dermo-palatine.

\section{OSTEOCRANIUM}

The development of the bony skull is quite varible in teleosts (De Beer, 1937; Bamford, 1948; Jollie, 1984; Matsurra \& Yomeda, 1987). In S. maximus, no ossified element is visible at hatching. This is true also in Clarias gariepinus, Barbus barbus, Chrysichthys auratus (Geoffroy Saint-Hilaire) (Family: Claroteidae), Heterobranchus longifilis, and Dicentrarchus labrax (Surlemont \& Vandewalle, 1991; Vandewalle et al., 1992, 1995, 1997; Gluckmann et al., unpublished). Galeichthys felis Valenciennes (Family: Bagridae) appears as an exception, since the dentaries, operculars, and premaxillaries are already present at hatching (Tilney \& Hecht, 1993). In Oncorhynchus mykiss Walbaum (Family: Salmonidae), an opercular, a maxillary, and a dentosplenial are present $24 \mathrm{~h}$ post-hatching (Verraes, 1977).

The first bones always seem to be of dermal origin in teleosts. In Esox lucius L. (Family: Esocidae), the first bones present are the maxillaries, followed by the premaxillaries (Jollie, 1975). In Lophius gastrophysus Libeiro (Family: Lophiidae), the maxillaries, premaxillaries, dentaries, operculars, and Suboperculars all appear simultaneously (Matsuura \& Yomeda, 1987). In Xenistius californiensis, the maxillaries appear first, followed by the pre-maxillaries and dentaries, and in Anisotremus davidsoni, the first bones in place are the premaxillaries, maxillaries, dentaries, preoperculars, and operculars (Watson \& Walker, 1992). In Nerophis aequoreus L. (Family: Syngnathidae), the first ossifications are a pair of maxillaries, a pair of premaxillaries, a pair of dentaries, a pair of angulars (Kadam, 1961). In Leuciscus rutilus, the pharyngeal jaws are the first to appear (Hubendick, 1942). In another cyprinid, Barbus barbus, the operculars, maxillaries, and dentaries develop first, followed by the lower pharyngeal jaws (fifth Ceratobranchials) (Vandewalle et al., 1992). In Chrysichthys auratus, the first in place are the dentaries, operculars, and two pairs of branchiostegal rays, followed by the maxillaries and operculars (Vandewalle et al., 1995a). Pharyngeal toothed plates will appear at the next stage, along with the first buccal teeth. Heterobranchus longifilis displays all at once the maxillaries, dentaries, premaxillaries, operculars, and upper and lower pharyngeal jaws (Vandewalle et al., 1997).

In three Silurus species, the maxillaries and premaxillaries come first (Kobayakawa, 1992). In Clarias gariepinus the opercular appears first (Surlemont \& Vandewalle, 1991), as in Oncorhynehus mykiss (Verraes, 1977), and is followed by the premaxillaries, maxillaries dentaries, and upper and lower pharyngeal toothed plates.

In S. maximus, the first bones appear on day 3. They belong to the dermal Splanchnocranium: they are the preoperculars. They are followed on day 8 by the maxillaries, dentaries, and operculars, on day 10 by toothed plates at the level of pharyngobranchials 3 and 4 , and on day 12 by the premaxillaries, angulo- 
articulars, pharyngobranchials 2, 3, and 4, and the fifth pair of Ceratobranchials. At this stage, the upper and lower pharyngeal jaws can be considered complete. As in Haplochromis elegans Trewawas (Family: Cichlidae), Lutjanus campechanus, Anisotremus davidsoni, Xenistius Californiensis, Centropomus undecimalis, and Dicentrarchus labrax, the maxillaries and premaxillaries are superposed at the outset. This feature is typical of species possessing a protractile mouth at the adult stage. There is thus no transition through a supposedly primitive state where the premaxillaries and maxillaries are adjacent. This latter situation occurs in Esox lucius and Oncorhynchus kisutch (Walbaum) (Family: Salmonidae) (Jollie, 1975, 1984). On day 13, the Suboperculars, interoperculars, ectopterygoids, and entopterygoids are present. On day 15, the first buccal teeth appear on the dentaries and premaxillaries, some $48 \mathrm{~h}$ after the appearance of the pharyngeal jaws.

The next bones to appear in $S$. maximus are the first enchondral ossifications of the Splanchnocranium. The hyomandibulars, symplectics, and quadrates develop simultaneously on day 18, after the dermal bones of the suspensorium, the entopterygoids, and the ectopterygoids. They are followed on day 19 by the palatines and on day 20 by the metapterygoids. While the development of the enchondral elements of the suspensorium can be considered similar in $\mathrm{S}$. maximus and Esox lucius (Jollie, 1975), it is different in other species. In Dicentrarchus labrax the quadrates, then the symplectics appear well before the entopterygoids and ectopterygoids; the hyomandibulars and palatines do not develop until later. In Lophius gastrophysus, the palatines and quadrates appear first, followed by the hyomandibulars, then the ectopterygoids and symplectics (Matsuura \& Yomeda, 1987). In Barbus barbus, the first are the quadrates; they are followed by the entopterygoids and hyomandibulars, then by the ectopterygoid and symplectics, and finally by the metapterygoids (Vandewalle et al., 1992). According to Kadam (1961), the quadrates appear at the same time as the dermal structures of the suspensorium in Neurophis aequoreus. There are thus similarities and differences between species in the timing of the development of enchondral elements of the suspensorium.

In S. maximus, ossification of the entopterygoid, ectopterygoid, palatine, metapterygoid, and Symplectic begins at the centre, while that of the quadrate and hyomandibular begins at the joint. This ossification pattern, which is common to many teleosts (Kadam, 1961; Jollie, 1975; Vandewalle et al., 1992, 1995b; Watson \& Walker, 1992; Potthoff \& Tellock, 1993; Gluckmann et al. (unpublished) suggested that the quadrato-mandibular and hyomandibulo-neurocranial joints are functionally important at an early stage.

The hyoid bars display four ossification centres in $S$. maximus: the hypohyals, the ceratohyal, the epihyal, and the interhyal. The first are the Ceratohyal and interhyal, appearing on day 19 , followed by the hypohyals and epihyal, visible on day 25. On day 19, ossification of the ceratohyal begins at the centre of the still-intact cartilaginous hyoid bar. Ossification of the interhyal begins in its central part. On day 20, the ceratohyal continues to ossify and replaces the central cartilage of the hyoid bar which by this time consists of only two small 
cartilaginous lumps, one on each side of the bony ceratohyal. On day 25, the ceratohyal has grown and surrounds the bericyform foramen. At this time, the bony epihyal appears at the site of the cartilaginous lump that extends the ceratohyal towards the rear. Finally, the two bony hypohyals, dorsal and ventral, form at the ends of the hypohyal cartilage, i.e. in the same cartilaginous mass. The dorsal hypohyal retains the foramen present in the hypohyal cartilage, the transhyoid foramen. The same timing is observed in the development of the hyoid arch, with the presence of a beryciform foramen in the ceratohyal, in Microspathodon chrysurus and Centropomus undecimalis (Potthoff et al., 1987; Potthoff \& Tellock, 1993), and without this foramen, in Lutjanus campechanus and Anisotremus davidsoni (Potthoff et al., 1988; Watson \& Walker, 1992).

Ossification of the branchial basket first affects the centres of the Ceratobranchials, then the centres of the epibranchials, and finally the centres of the pharyngobranchials. The hypobranchials and axes of the basibranchials ossify last. The first pair of Ceratobranchials and the three pairs of pharyngobranchials bear upper and lower pharyngeal toothed plates even before they ossify. In other acanthoptergians, specifically Lutjanus campechanus, Anisotremus davidsoni, Xenistius Californiensis, and Centropomus undecimalis, all five ceratobranchials appear together, at the same time as the first pharygneal toothed plates, and are followed by the epibranchials, pharyngobranchials, and hypobranchials; then finally, the axes of the basibranchials ossify (Potthoff et al., 1988; Watson \& Walker, 1992; Potthoff \& Tellock, 1993). In Dicentrarchus labrax, the first four Ceratobranchials ossify at the time the upper toothed plates appear, the bony fifth Ceratobranchials appear shortly afterward, before the rest of the branchial basket (Gluckmann \& Vandewalle, unpublished). Among Ostariophysi, ossification of the branchial basket is variable, but the pharyngeal jaws and their teeth appear very early in development before the other branchial elements (Surlement \& Vandewalle, 1991; Vandewalle et al., 1992, 1995a, 1997).

In S. maximus, the neurocranium tends to develop more slowly than the Splanchnocranium, a usual phenomenon in teleosts (De Beer, 1937; Vandewalle et al., 1992, 1995a). The bony structures appear in a different chronological order than generally observed. The frontals appear at an exceptionally early stage, well before the parasphenoid.

In other species such as Oncorhynchus mykiss, Esox lucius, Clarias gariepinus, Barbus barbus, Anisotremus davidsoni, and Xenistius Californiensis, the parasphenoid is the first bony structure of the neurocranium to appear, before formation of the frontals (Jollie, 1975; Verraes, 1977; Surlemont \& Vandewalle, 1991; Vandewalle et al., 1992; Watson \& Walker, 1992). In S. maximus, the parasphenoid, independent of the trabecular bars, does not appear until day 13 and it will constitute the braincase floor. The following steps see the appearance of a basioccipital on day 15 , followed by two exoccipitals, two parietals, and two pterotics on day 18 . On day 19 , no bony structure yet closes the rear of the braincase dome. The successive appearance of the basioccipital and exoccipitals is also observed in Clarias gariepinus, Barbus barbus, Anisotremus davidsoni, and Xenistius Californiensis (Surlemont \& Vandewalle, 1991; Vandewalle et al., 1992; Watson \& Walker, 1992). In 
Nerophis aequoreus and Esox lucius, the exoccipitals and basioccipital appear simultaneously (Kadam, 1961; Jollie, 1975). The late and simultaneous development of several neurocranial structures such as the lateral ethmoids, sphenotics, prootics, epiotics, pleurosphenoids, and Supraoccipital is not specific to S. maximus. In Dicentrarchus labrax the Supraoccipitals, pterotics, sphenotics, and frontals appear simultaneously on day 30 (Gluckmann et al., unpublished); they appear all at once on day 24 in Barbus barbus (Vandewalle et al., 1992) and on day 15 in Chrysichthys auratus (Vandewalle et al., 1995).

By comparing alcyan-blue-stained and alizarin-stained specimens at the same developmental stage, it is easy to see that the ossifications of the deeper skeleton appear where cartilage regression is about to occur. In this situation what actually takes place is not a regression but a resorption of the cartilage in favour of ossification. Regression sensu stricto affects only the trabecula communis, the taeniae marginales, and Meckel's cartilages. The trabecula communis begins to shrink and to separate from the braincase on day 15, the left and right taeniae marginales begin to regress on day 23 and are distinct from the lamina precerebralis on day 29; Meckel's cartilages are reduced on day 10 to two cartilaginous lumps in front of the lower jaws. Resorption concerns, for example, the Ceratobranchials and hyoid bars.

Ossification may begin before resorption or at the same time. In S. maximus, this correspondence is easy to see at the level of the Ceratobranchials, hyoid bars, and parts of the suspensorium. For instance, ossification is visible at the centres of the Ceratobranchials on day 17, before resorption of these structures is obvious on day 18. At this same stage (day 18), the quadrate and hyosymplectic start to ossify before resorption of the corresponding cartilages is initiated (palatoquadrates) or when it has just begun (hyosymplectics).

\section{GENERAL CONSIDERATIONS}

In S. maximus, as in Centropomus Undecimalis and Dicentrarchus labrax (Potthoff \& Tellock, 1993; Gluckmann et al., unpublished), no skeletal structure is present at hatching. In these species, the cartilaginous structures of the head develop progressively from the first day. In some other species, the first structures are present before hatching (DeBeer, 1937).

It seems impossible to define a model of Chondrocranial development in teleosts, so great are the variations in the chronology of appearance of the various structures. Similarities between species are distinguishable, however. This is the case for Dicentrarchus labrax and $S$. maximus. In both species, the cartilaginous neurocranium develops faster posteriorly than anteriorly. Both notably lack a basal plate. They display similar timing in the appearance of the elements forming the hyoid arch. In both, the pharyngobranchials appear progressively from rear to front. Both display cartilage resorption before the chondrocranium is finished, affecting first the trabecular bars, then the Ceratobranchials and hyoid bars. Lastly, in both species, resorption of Meckel's cartilages, isolates two small cartilaginous lumps at the front of the mandible. These similarities suggest a relationship between a pleuronectiform and a 
perciform, these orders being currently associated through their classification as percomorphs (Nelson, 1994). A more surprising observation is that the anguiliform Anguilla anguilla also shows similarities to $S$. maximus: a tropibasictype skull, the absence of an epiphyseal bridge defining a single large fontanelle, a basihyal independent of the basibranchials, and resorption of the taeniae marginales during an original metamorphosis.

Whatever the variations observed in the postembryonic development of teleosts, the manner in which the skull develops must meet the survival requirements of the fry. At hatching, in S. maximus as well as other teleosts, the well-vascularized yolk sac performs both a nutritional and a respiratory function.

In S. maximus fry, feeding is essentially endogenous up to day 2 post-hatching (Cousin \& Laurencin, 1985). Between days 2 and 3 post-hatching, the mouth opens and mixed-type feeding begins. From this time on, the digestive tube differentiates and becomes functional with a capacity for digestion and intestinal absorption (Cousin \& Laurencin, 1985). By day 7 post-hatching, feeding is totally exogenous (Cousin \& Laurencin, 1985). The stomach undergoes changes: major muscular differentiation of the fundal and cardiac parts, and the start of gastric gland formation (Cousin \& Laurencin, 1985). In S. maximus, as the yolk sac regresses, the branchial respiratory system develops. The first four branchial arches, bearing respiratory filaments, are present on day 3 and can carry out their function which is compatible with a cartilaginous skeleton. The fifth Ceratobranchials and pharyngobranchials 2, 3, and 4, needed for ingesting and processing food, do not bear dermal toothed plates until days 10 and 12 . The buccal parts are not complete until day 12 and do not bear teeth until day 15 .

The parasphenoid, lastly, does not appear until day 13; which is long after exogenous feeding begins. In many teleosts, the parasphenoid and the bony pharyngeal and buccal jaws appear before exogenous feeding begins or at the same time (Potthoff et al., 1987, 1988; Surlemont \& Vandewalle, 1991; Vandewalle et al., 1992, 1995a, 1997; Watson \& Walker, 1992; Gluckmann et al., unpublished). By closing at least partially the hypophyseal fenestra, the parasphenoid isolates the braincase from the buccal cavity and makes food ingestion possible without any impact on the brain. In $S$. maximus, however, the absence of a parasphenoid at the time of weaning from the yolk sac probably does not constitute an obstacle to the ingestion of hard food because S. maximus displays no hypophyseal fenestra and the trabecula communis is probably adequate for isolating the braincase. The food of farm-reared turbots consists, according to Person-Le-Ruyet et al. (1989), of rotifers $(250 \mu \mathrm{m})$ and of Artemia sp. nauplii $(500 \mu \mathrm{m})$. On day 8 , the fry is equipped with dermal bones such as a dentary, a maxillary, a small opercular, and with flexible, elastic cartilaginous elements such as a hyosymplectic articulating with the neurocranium, a palatoquadrate articulating with Meckel's cartilage, and a hyoid bar. These structures are doubtless capable of performing movements that create a water current from front to rear (Vandewalle \& Chardon, 1981; Lauder, 1985), probably a fairly inefficient one but sufficient for sucking food into the buccal cavity. On day 13 , the fry has tooth-bearing pharyngeal jaws and toothless buccal jaw rendering it more apt at seizing and processing prey such as $500-\mu m$ 
Artemia nauplii and later 1000- $\mathrm{m}$ metanauplii (Person-Le-Ruyet et al., 1989) The shape and arrangement of the buccal parts even suggest that the upper jaws might extend (Eaton, 1935; Liem, 1970, 1978; Lauder \& Liem, 1981; Lauder \& Shaffer, 1993; Vandewalle et al., 1995a), thus creating a prebuccal cavity like that of adult acantopterygians, which improves the sucking system (Vandewalle \& Chardon, 1981; Motta, 1984; Westneat \& Wainwright, 1989). This deployment mechanism might be influenced by the asymmetries appearing at metamorphosis According to Van Dobben (1935) and Yazdani (1969), the left and right upper jaws of the $S$. maximus adult might protract asymmetrically due to rightward leaning of the mesethmoid's median crest and to the presence of a ligament connecting the palatine to the ethmoid cartilage (situated below the ascending processes of the premaxillaries) on the blind side. When the mouth opens, the ethmoid cartilage does in fact slide along the mesethmoid, which should turn it towards the blind side, and the ligament connecting the palatine to the ethmoid cartilage should, upon extending, have the same effect. This deviation would be neutralized by the difference in length between the left and right upper jaws. The results of the present work can neither confirm nor invalidate Van Dobben's (1935) and Yazdani's (1969) hypotheses, because the asymmetries of the upper jaws present in the fry are still too minor.

Metamorphosis hardly seems to affect the cartilaginous skeleton in $S$. maximus. The only consequence of sinistral torsion and migration of the right eye is the early resorption of the right taenia marginalis just before metamorphosis. The left taenia marginalis is not resorbed until much later.

This is the only observed asymmetry in the development and evolution of the cartilaginous cephalic skeleton in S. maximus. The buccal parts, notably, display no asymmetry, even though they do in the adult. The absence of an epiphyseal bridge, the early resorption of the tectum synoticum and taenia tecta medialis, must doubtless be related to metamorphosis. Once resorbed, these structures no longer represent an obstacle to migration of the right eye.

While metamorphosis has little bearing on the cartilaginous skeleton, it produces asymmetries in the bony skeleton (suspensorium, jaws, front of the neurocranium). The asymmetries affecting the frontals and lateral ethmoids are among the most striking. When they appear, the frontals are symmetrical; they deform progressively in the course of metamorphosis. The early formation of the frontals probably reflects the need to reinforce the connection between the front and back of the braincase, an imperative rendered all the more important by the fact that on day 23 , the right taenia marginalis separates from the lamina orbitonasalis and thus no longer constitutes the solid frame of the orbital region. Well-developed frontals might offset this separation, related to migration of the right eye to the left side. Likewise, the difference between the two lateral ethmoids, described here in detail, increases as metamorphosis progresses, the right lateral ethmoid growing to support the migrating eye (Brewster, 1987).

Yet other functional imperatives are liable to influence the construction of the cephalic skeleton. For instance, the late closure of the dome and lateral walls of the braincase might reflect necessities linked with growth of the brain. Likewise, 
even though the late appearance of some cephalic structures such as the lateral ethmoids, nasals, and mesethmoid does not seem to influence fry survival, these structures are doubtless subject to constraints as important as those which determine the development of the earlier-appearing structures.

The authors thank M. Leclercq and Mme. Lebègue (France Turbot-NATA), for rearing and selecting the specimens studied. FW is a researcher at the Fonds pour la Recherche dans l'Industrie et l'Agriculture.

\section{References}

Badenhorst, A. (1989a). Development of the chondrocranium of the shallow-water cape hake Merluccius capensis (Cost.). Part 1: neurocranium. South African Journal of Zoology 24, 33-48.

Badenhorst, À. (1989b). Development of the chondrocranium of the shallow-water cape hake Merluccius capensis (Cost.). Part 2: viscerocranium. South African Journal of Zoology 24, 49-57.

Bamford, T. W. (1948). Cranial development of Galeichthys felis. Proceedings of Zoological Society, London 118, 364-391.

Berrill. N. J. (1925). The development of the skull in the sole and the plaice. Quarterly Journal of Microscopical Science 69, 217-244.

Bertmar, G. (1959). On the ontogeny of the chondral skull in Characidae. with a discussion on the Chondrocranial base and visceral chondrocranium in fishes. Acta Zoologica, Stockholm 40, 203-364.

Bhargava, H. N. (1958). The development of the chondrocranium of Mastacemhelus armatus (Cuv. et Val.). Journal of Morphology 102, 401-426.

Blaxter, J. H. S., Danielssen, D., Moksness, E. \& Øiestad, V. (1983). Description of the early development of the halibut Hippoglossus Mppoglossus and attempts to rear the larvae past first feeding. Marine Biology 73, 99-107.

Brewster, B. (1987). Eye migration and cranial development during flatfish metamorphosis: a reappraisal (Teleostei: Pleuronectiformes). Journal of Fish Biology 31, 805-833.

Cousin, J. C. B. \& Laurencin, F. B. (1985). Morphogénèse de l'appareil digestif et de la vessie gazeuse du turbot, Scophthalmus maximus. Aquaculture 47, 305-319.

Daget, J. (1959). Note sur les Distichodus (Poissons Characiformes) de IOuest africain. Bulletin de l'Institut français d'Afrique noire, sèrie A 21, 1275-1303.

Daget, J. (1964). Le crâne des téléostéens. Mémoires du Museum national d'Histoire naturelle, série A, Zoologie 31, 167-340. 
Daget, J. \& d'Aubenton, F. (1957). Développement et morphologie du crâne d'Heterotis Mloticus Ehrenberg. Bulletin de l'Institut français d'Afrique noire, série A 19, 881-936.

Daget, J. \& d'Aubenton, F. (1960). Morphologie du chondrocrâne de Mormyrus rume. Bulletin de l'Institut français d'Afrique noire, série A 22, 1013-1052.

De Beer, G. R. (1937). The Development of the Vertebrate Skull. Oxford: Clarendon Press.

Dingerkus, G. \& Uhler, L. D. (1977). Enzyme clearing of alcyan blue stained whole small vertebrates for demonstration of cartilage. Stain technology 52, 229-232.

Dobben, van, H. W. (1935). Über den Kierfermechanismus der Knochenfische. Archives néerlandaises de Zoologie 2, 1-72.

Eaton, T. H. (1935). Evolution of the upper jaw mechanisms in teleost fishes. Journal of Morphology 58, 157-172.

Elman, J. F. \& Balon, E. K. (1980). Early ontogeny of white sucker Catostomus Commersonii, with steps of saltatory development. Environmental Biology of Fishes 5, 191-224.

Gaupp, E. (1903). Zür Entwicklung der Schadelknocken bei den Teleostieren. Verhandlungen der anatomischen Gesellschaft, lena 1903, 113-123.

Hubendick, B. (1942). Zür Kenntnis der Entwicklung des Primordialcraniums bei Leuciscus rutilus. Arkiv für Zoologi 34A, 1-35.

Jollie, M. (1975). Development of the head skeleton and pectoral girdle in Esox. Journal of Morphology 147, 61-88.

Jollie, M. (1984). Development of the head skeleton and pectoral girdle of salmons, with a note on the scales. Canadian Journal of Zoology 62, 1757-1778.

Kadam, K. M. ( 1961). The development of the skull in Neurophis (Lophobranchii). Acta Zoologica 42, 1-42.

Kindred, J. E. (1919). Development of skull in Ameiurus nebulosus. Illinois Biology Monographs 5, 7-121.

Kobayakawa, M. (1992). Comparative morphology and development of bony elements in the head region in three species of Japanese catfishs (Silurus: Siluridae: Siluriformes). Japanese Journal of Ichthyology 39, 25-36.

Krupka, I. (1988). Early development of the barbel Barbus barbus (Linnaeus, 1758). Prace ustavu rybarstva a hydrobiologica 6, 115-138.

Lagardère, F., Boulhic, M. \& Bürgin, T. (1993). Anomalies in the cephalic area of laboratory-reared larvae and juveniles of the common sole, Solea solea: oral jaw apparatus, dermal papillae and pigmentation. Environmental Biology of Fishes 36 3546. 
Lauder, G. V. (1985). Aquatic feeding in lower vertebrates. In Functional Vertebrate Morphology (Hildebrand, M., Bramble, D. M., Liem, K. F. \& Wake, D. B., eds), pp. 210-229. Cambridge: Harvard University Press.

Lauder, G. V. \& Liem, K. F. (1981). Prey capture by Luciocephalus pulcher: implications for models of jaw protrusion in teleost fishes. Environmental Biology of Fishes $\mathbf{6}$, 257-268.

Lauder, G. V. \& Shaffer, H. B. (1993). Design of feeding systems in aquatic vertebrates: major patterns and their evolutionary interpretations. In The Skull Vol. 3, (Hanken, J. \& Hall, B. K., eds), pp. 113-149. Chicago: University of Chicago Press.

Liem, K. F. (1970). Comparative functional anatomy of the Nandidae (Pisces: Teleostei). Fieldiana Zoology 56, 1-166.

Liem, K. F. (1978). Modulatory multiplicity in the functional repertoire of the feeding mechanism in cichlid fishes. I. Piscivores. Journal of Morphology 158, 323-360.

Liew, H.-C., Milward, N. E. \& Hartwick, R. F. (1988). Descriptions of larval flatfishes of the genera Psettodes (Psettodidae) and Pseudorhombus (Paralichthyidae) from the Great Barrier Reef, Australia. Australian Journal of Marine and Freshwater Research 39, 51-70.

Matsuura, Y. \& Yomeda, N. T. (1987). Osteological development of the lophiid anglerfish, Lophius gastrophysus. Japanese Journal of Ichthyology 33, 360-367.

McAllister, D. E. (1968). The evolution of branchiostegals and associated opercular, angular and hyoid bones and the classification of teleostome fishes, living and fossil. Bulletin of the National Museum of Canada 221, 1-239.

Motta, P. J. (1984). The mechanism and functions of jaw protrusion in teleost fishes: a review. Copiea 1984, 1-18.

Nelson, J. S. (1994). Fishes of the World. Chichester: John Wiley.

Norman, J. R. (1926). The development of the chondrocranium of the eel (Anguilla vulgaris) with observations on the comparative morphology and development of the chondrocranium in bony fishes. Philosophical Transactions of the Royal Society of London B 214, 369-464.

Parker, W. K. (1873). On the structure and development of the skull in salmon (Salmo salar). Philosophical Transactions of the Royal Society of London 163, 95-145.

Person-Le-Ruyet, J., Lahaye, J., Deniel, C., Metailler, R., Devauchelle, N., Menu. B., Noel, T. \& Baudin-Laurencin, F. (1989). L'élèvage des poissons plats: sole, turbot. In Aquaculture, Vol. 2 (Barnabé, G., coord.), pp. 721-775. Paris: Technique et Documentation Lavoisier. 
Potthoff, T. (1983). Clearing and staining techniques. American Society of Ichthyologists and Herpetologists, Special Publication 1, 35-37.

Potthoff, T. \& Tellock, J. A. (1993). Osteological development of the snook, Centropomus Undecimalis (Teleostei, Centropomidae). Bulletin of Marine Science 52, 669-716.

Potthoff, T., Kelley, S., Saksena, V., Moe, M. \& Young, F. (1987). Description of larval and juvenile yellowtail damselfish Microspathodon chrysurus, Pomacentridae, and their osteological development. Bulletin of Marine Science 40, 330-375.

Potthoff, T., Kelley, S. \& Collins, L. A. (1988). Osteological development of the red snapper, Lutjanus Campechanus (Lutjanidae). Bulletin of Marine Science 43, 1-40.

Sadiq, J. \& Gibson, R. (1984). The developmental stages of larval turbot, Scophthalmus maximus (L.). The Journal of Experimental Marine Biology and Ecology 82, 35-51.

Srinivasachar, H. R. (1959). Development of the skull in catfishes: part III: the development of the chondrocranium in Heteropneustes fossilis (Bloch) (Heteropneustidae) and Clarias batrachus (Linn.) (Clariidae). Morphologisches Jahrbuch 101, 373-405.

Stöhr, P. (1882). Zür Entwicklungsgeschichte des Kopfskelettes der Teleostier. In Festchrift 3 säkulafeier Alma Julia Maximiliana. Universitats Wunzburg, Leipzig 2, $1-23$.

Surlemont, C. \& Vandewalle, P. (1991). Développement postembryonnaire du squelette et de la musculature de la tête de Clarias gariepinus (Pisces, Siluriformes) depuis l'éclosion jusqu'à 6.8 mm. Canadian Journal of Zoology 69, 1094-1103.

Swinnerton, H. H. (1902). A contribution to the morphology of the teleostean head skeleton, based upon a study of the developing skull of the three-spined stickleback (Gasterosteus aculeatus). Quarterly Journal of Microscopical Sciences 45, 503-593.

Taylor, W. R. \& Van Dyke, G. C. (1985). Revised procedures for staining and clearing small fishes and other vertebrates for bone and cartilage study. Cybium 9, 107-121.

Tilney, R. L. \& Hecht, T. (1993). Early ontogeny of Galeichthys feliceps from the south east coast of South Africa. Journal of Fish Biology 43, 183-212.

Tischomiroff, A. (1885). Zfir Entwicklung des Schadels bei der Teleostieren. Zoologischer Anzeiger 8, 533-537.

Vandewalle, P. \& Chardon, M. (1981). Réflexions sur les rapports entre forme, structure et fonction chez des poissons de la famille des Cyprinidae. Cybium 5, 67-70.

Vandewalle, P., Focant, B., Huriaux, F. \& Chardon, M. (1992). Early development of the cephalic skeleton of Barbus barbus (Teleostei, Cyprinidae). Journal of Fish Biology 41, 43-62. 
Vandewalle, P., Laleye, P. \& Focant, B. (1995a). Early development of cephalic bony elements in Chrysichthys auratus (Pisces, Siluriformes, Bagriidae). Belgian Journal of Zoology 125, 329-347.

Vandewalle, P., Saintin. P. \& Chardon, M. (1995b). Structures and movements of the buccal and pharyngeal jaws in relation to feeding in Diplodus sargus. Journal of Fish Biology 46, 623-656.

Vandewalle, P., Gluckmann, L, Baras, E., Huriaux, F. \& Focant, B. (1997). Postembryonic development of the cephalic region in Heterobranchus Iongifilis. Journal of Fish Biology 50, 227-253.

Verraes, W. (1977). Postembryonic ontogeny and functional anatomy of the ligamentum mandibulo-hyoideum and the ligamentum interoperculo-mandibulare, with notes on the opercular bone and some other cranial elements in Salmo gairdneri Richardson, 1836 (Teleostei: Salmonidae). Journal of Morphology 151, 111-119.

Watson, W. \& Walker, Jr, H. J. (1992). Larval development of sargo (Anisotremus davidsoni) and salema (Xenistius Californiensis) (Pisces, Haemulidae) from the Southern California bight. Bulletin of Marine Science 51, 360-406.

Weastneat, M. W. \& Wainwright, P. C. (1989). Feeding mechanism of Epibulus insidiator (Labridae, Teleostei): evolution of a novel functional system. Journal of Morphology 202, 129-150.

Wells, F. R. (1923). On the morphology of the chondrocranium of the larval herring (Clupea harengus). Proceedings of the Zoological Society of London 92, 1213-1229

Williams, S. R. (1902). Changes accompanying the migration of the eye and observations on the tractus opticus and tectum opticum in Pseudopleuronectes americanus Bulletin of the Museum of Comparative Zoology, Harvard 40, 1-57.

Winslow, G. W. (1897). The chondrocranium of the Ichtiopsida. Tufts College Studies 5 $147-201$.

Yazdani, G. M. (1969). Adaptation in the jaws of flatfish (Pleuronectiformes). Journal of Zoology 159, 181-222. 